\title{
Trabecular variation in the first metacarpal and manipulation in hominids.
}

\author{
Christopher J. Dunmore ${ }^{1}$, Ameline Bardo ${ }^{1}$, Matthew M. Skinner ${ }^{1,2}$, and Tracy L. Kivell ${ }^{1,2}$, \\ ${ }^{1}$ Skeletal Biology Research Centre, School of Anthropology and Conservation, University of Kent \\ ${ }^{2}$ Department of Human Evolution, Max Planck Institute for Evolutionary Anthropology
}

\section{Abstract}

\section{Objectives}

The dexterity of fossil hominins is often inferred by assessing the comparative manual anatomy and behaviors of extant hominids, with a focus on the thumb. The aim of this study is to test whether trabecular structure is consistent with what is currently known about habitually loaded thumb postures across extant hominids.

\section{Materials and Methods}

We analyse first metacarpal (Mc1) subarticular trabecular architecture in humans (Homo sapiens, $\mathrm{n}=10$ ), bonobos (Pan paniscus, $\mathrm{n}=10$ ), chimpanzees (Pan troglodytes, $\mathrm{n}=11$ ), as well as for the first time, gorillas (Gorilla gorilla gorilla, $\mathrm{n}=10$ ) and orangutans (Pongo sp., $\mathrm{n}=1$, Pongo abelii, $\mathrm{n}=3$ and Pongo pygmaeus, $\mathrm{n}=5$ ). Using a combination of subarticular and whole-epiphysis approaches, we test for significant differences in relative trabecular bone volume (RBV/TV) and degree of anisotropy (DA) between species.

\section{Results}

Humans have significantly greater RBV/TV on the radio-palmar aspects of both the proximal and distal Mc1 subarticular surfaces and greater DA throughout the Mc1 head than other hominids. Nonhuman great apes have greatest RBV/TV on the ulnar aspect of the Mc1 head and the palmar aspect of the Mc1 base. Gorillas possessed significantly lower DA in the Mc1 head than any other taxon in our sample.

\section{Discussion}

These results are consistent with abduction of the thumb during forceful 'pad-to-pad' precision grips in humans and, in non-human great apes, a habitually adducted thumb that is typically used in precision and power grips. This comparative context will help infer habitual manipulative and locomotor grips in fossil hominins.

\section{Introduction}

The unique dexterity of the human hand is often linked to two major events in hominin evolution, the development of obligate bipedalism and of complex technology (Wood-Jones, 1916; Napier, 1993; Marzke, 2013; Lemelin \& Schimtt, 2016; Richmond, Roach \& Ostrofsky, 2016). The discovery in the late 1950s of stone tools in association with the $\mathrm{OH} 7 \mathrm{Homo}$ habilis fossil hand, dated to approximately 1.75 million years ago (Ma), was interpreted as potential anatomical and behavioural evidence of human-like dexterity (Napier, 1962; Leakey, Tobias \& Napier, 1964; de la Torre, 2011). Archaeological evidence of hominin tool behaviours has since been found in earlier contexts, dating back to at least 3.3 $\mathrm{Ma}$ (Harmand et al., 2015), and is likely a preserved facet of a larger hominin manipulative repertoire that may have older origins (Panger, Brooks, Richmond \& Wood, 2002; 
Haslam et al., 2009; Alba Moyà-Solà \& Köhler, 2003; Kivell, 2015). When and how the manipulative capability required for stone tool behaviours evolved, however, is still a key question in human evolution (Panger et al., 2002; Richmond et al., 2016).

As our closest living relatives, the manipulative abilities of hominid hands have been used to functionally interpret fossil hand bones (e.g. Susman, 1994; 1998; Marzke, 1997). Napier's (1956; 1993) broad schema of power grips, usually practised by apes in locomotion, and precision grips, generally practised by humans during manipulation, provides an intuitive dichotomy of phylogenetic polarity that has been the basis for this functional inference. Ape-like aspects of fossil hominin hand morphology are often interpreted as useful for arboreal locomotion whereas human-like morphological features are interpreted as advantageous for manipulation (e.g., Susman, 1994; Tocheri, Orr, Jacofsky \& Marzke, 2008; Marzke, 2013; Kivell et al.,2011; 2015). In particular, compared to other apes, humans possess a relatively long thumb with a robust first metacarpal (Mc1) and broad phalanges that have been interpreted as key to enabling forceful 'pad-to-pad' precision grips. Forceful precision grips have been traditionally considered unique to humans (Marzke \& Wullstein, 1996) and facilitate stone tool production (Marzke \& Shackley, 1986; Marzke et al., 1998, Key \& Dunmore, 2015) and use (Williams-Hatala et al., 2018; Key, Merritt \& Kivell, 2018). Here, rather than external shape or size, we analyse another aspect of the Mc1 morphology across great apes, the internal trabecular architecture.

Internal trabecular structure can provide additional evidence of how a bone was loaded during life, rather than the limits of joint movement its external shape permits (Ruff \& Runstead, 1992; Currey, 2002), and thus potentially provide novel insight into fossil hominin hand use. Biomechanical loading causes trabeculae to remodel, a process known as bone functional adaptation (Cowin, 1986; Frost, 1987; Ruff, Holt and Trinkhaus, 2006). Though trabecular bone, like all bone, is to some extent heritable (Havill et al., 2010) and is perhaps most responsive early in ontogeny (Lovejoy, McCollum, Reno \& Rosenman, 2003; Wallace, Demes \& Judex, 2017), a variety of non-primate taxa have demonstrated trabecular bone functional adaptation in controlled experimental conditions (Biewener, Fazzalari, Konieczynski, \& Baudinette, 1996; Pontzer et al., 2006; Barak, Lieberman \& Hublin, 2011; Christen \& Muller, 2017). This trabecular functional adaptation is most often experimentally demonstrated by increased trabecular bone volume (BV/TV) and increased alignment of trabeculae (degree of anisotropy, DA) in the direction of a novel load (Pontzer et al., 2006; Barak, Lieberman \& Hublin, 2011). The combination of a higher BV/TV and orientation of trabeculae to the principle axis of load, can explain up to $92 \%$ of variation in the biomechanical properties of bone (Ulrich, Van Rietbergen, Laib \& Ruegsegger, 1999; Lambers et al., 2013).

Trabecular studies of primate hands have also found that the trabecular architecture is consistent with hand positions thought to be used by different species during locomotion (Zeininger, Richmond \& Hartman, 2011; Tsegai et al. 2013; Chirchir, Zeininger, Nakatsukasa, Ketcham \& Richmond, 2017, Barak, Sherratt, \& Lieberman, 2017; Dunmore, Kivell , Bardo \& Skinner, 2019). Many of these studies have focussed on the metacarpal heads, likely due to the biaxial movements afforded at the metacarpophalangeal joints as well as their proximity to prehensile and locomotor substrates, which together allow for relatively straight-forward functional interpretation of trabecular morphology. Further trabecular bone architecture has been shown to correlate with grip strength in some human hand bones, including the trapezium (Reina, Cavaignac, Trousdale, Laffosse \& Braga, 2017). Indeed the region of the trapeziometacarpal joint in which osteoarthritis first develops, thought to 
therefore endure the most substantial habitual load (Koff et al., 2003) is also the area in which trabecular bone is densest on the Mc1 (Stephens, Kivell, Pahr, Hublin \& Skinner, 2018). Preserved trabeculae in fossil hominins have been used to infer habitual loading and reconstruct locomotor (e.g. DeSilva \& Devlin, 2012; Barak et al., 2013; Su, Wallace \& Nakatsukasa, 2013; Zeininger, Patel, Zipfel \& Carlson, K. J, 2016; Ryan et al., 2018) and manipulative (e.g. Skinner et al., 2015; Stephens et al., 2018) behaviours during human evolution. These functional inferences are based on the comparative context of extant great apes and an association between variation in their trabecular architecture and assumptions about the joint postures they most commonly use (Orr, 2016).

Studies of trabeculae in the thumb have mainly focussed on humans. Right human Mc1s have a significantly greater BV/TV than those from left hands (Stephens et al., 2016), consistent with crosscultural right-hand bias in our species (Faurie, Schiefenhvel, leBomin, Billiard \& Raymond, 2005; Reina et al.,2017) though the trabecular difference was small in absolute terms (Skinner et al., 2015; Stephens et al., 2016; Reina et al., 2017). While BV/TV is significantly greater in the Mc1 head of both humans and chimpanzees relative to the base (Lazenby, Skinner, Hublin \& Boesch, 2011; Stephens et al. 2016) the species differ in the Mc1 base. Specifically the human Mc1 base has a greater concentration of trabecular bone in its palmar aspect relative to human non-pollical metacarpals (Wong, Meals \& Ruff, 2018) and the Mc1 of Pan (Skinner et al., 2015). Where Skinner et al. (2015) inferred function by qualitatively analysing 3D trabecular models, Stephens et al. (2018) quantitatively analysed an expanded sample of foragers and post-Neolithic humans and found that BV/TV was greatest in the radio-palmar segments of the Mc1 head and base, consistent with a flexed, abducted thumb in precision grips. This study also found that DA was lower in the Mc1 base of foragers relative to a sample of post-Neolithic humans, and related this to more varied loading at this, and other manual, joints in the forager population (Stephens et al., 2018).

We build on this work by analysing Mc1 trabeculae across extant hominids including modern humans (Homo sapiens), bonobos (Pan paniscus), chimpanzees (Pan troglodytes verus), as well as for the first time, gorillas (Gorilla gorilla gorilla) and orangutans (Pongo abelii and Pongo pygmaeus). A geometric morphometric, statistical mapping method (Dunmore et al., 2019) is applied to subarticular regions of trabecular models produced by the whole-epiphysis approach. We measure and statistically analyse variation in relative trabecular bone volume (RBV/TV; see below) and DA in the proximal and distal Mc1. The distribution of trabecular volume and level of alignment represented by these variables reflect the ability of the whole bone to resist load in different directions and so should be consistent with habitual thumb loading postures in these species.

\section{Locomotion, manipulation and thumb morphology}

Trapeziometacarpal (TMC) and metacarpophalangeal (MCP) joint movement and loading is a complex product of both bony and soft tissue morphology (van Leeuwen, Vanhoof, Kerkhof, Stevens \& Vereecke, 2018) and compared to humans little is known of actual loads experienced by the nonhuman great ape thumb, during locomotion or manipulation (Samuel, Nauwelaerts, Stevens \& Kivell, 2018). However, qualitative observations of force, which was judged by how apparently resistant objects were to the grip applied, during food processing do exist for some species (Marzke, Marchant, McGrew \& Reece, 2015; Neufuss, Robbins, Baeume, Hulme \& Kivell,2018). Further, combining what is known of this morphology with observed habitual thumb use allows for the broad characterisation of habitual thumb postures that are loaded in the species studied. 


\section{Homo sapiens}

Humans are obligate bipeds and so rarely employ grips in locomotion but power grips are used to habitually climb in some populations (Kraft, Venkataraman \& Dominy, 2014). The uniquely human power-squeeze grip is also used in manipulation (Marzke, Wullstein \& Viegas, 1992; Key, Merritt, \& Kivell, 2018). This grip flexes fingers around a cylindrical object, which diagonally lies across the palm, while the thumb is adducted with considerable force and controls the direction in which force is applied to the object (Cooney \& Chao, 1977; Marzke et al., 1992).

Human precision grips can also be uniquely forceful as demonstrated by the relatively high levels of pressure on the distal thumb of both hands during stone tool production and use (Key \& Dunmore, 2015; Williams-Hatala et al., 2018). During stone tool production, a 'three-jaw-chuck' grip is commonly used to wield hammerstones, in which the thumb is abducted and rotated to oppose the second and third digits (Markze, 1997). When using small flake stone tools, humans tend to use 'pad-to-side' grips whereas for larger flakes or handaxes they often employ a 'cradle' or 'five-jaw buttressed pad-to-pad power grip, which both oppose the thumb to the other fingers with support from the palm (Rolian, Lieberman \& Zermeno, 2011; Key et al., 2018). Biomechanical analysis has also shown large pollical flexion forces are required to stabilise a simulated tool during use (Rolian et al., 2011). While the role of the flexor pollicis longus muscle is debated (Hamrick, Churchill, Schmitt \& Hylander, 1998; Marzke et al., 1998), electromyography (EMG) data has highlighted that flexor pollicis brevis and opponens pollicis are strongly recruited to oppose the thumb to the rest of the fingers in these strong precision grips (Marzke et al., 1998). Clinical EMG studies have also demonstrated that the human opponens pollicis and abductor pollicis brevis muscles are highly recruited in opposition of the thumb during a pad-to-pad grip where they were not as highly recruited in a 'pad to side-grip' (Johanson, Valero-Cuevas \& Hentz, 2001).

Humans possess the longest thumb relative to the fingers among hominids (Almécija, Smaers \& Jungers, 2015), which facilitates opposition of thumb to the fingers (Napier, 1956; Marzke, 1997; Feix, Kivell, Pouydebat \& Dollar, 2015; Bardo, Vigouroux, Kivell \& Pouydebat, 2018). Human distal phalanges are capable of passive hyperextension as our deep flexor tendons are long compared to those of other great apes (Preuschoft, 1965; Tuttle, 1967). This movement permits full pad-to-pad precision grips (Napier, 1960) frequently used by humans to forcefully manipulate small objects, especially within the hand (Christel, 1993; Marzke \& Wullstein, 1996; Bardo, Cornette, Borel \& Pouydebat, 2017; Key et al., 2018). Humans are unique among hominids in possessing an extensor pollicis brevis muscle, which is well-developed and stabilises the extended McP joint while the first interphalangeal joint is forcefully flexed, as well as abducting the thumb (Marzke et al., 1999; Diogo, Richmond \& Wood, 2012). Indeed, while flexion at the McP joint in great apes appears to be limited to $90^{\circ}$ in non-human great apes due to their thenar eminence (Tuttle, 1969), clinical data suggests human McP joint flexion is limited to just $70^{\circ}$ in humans (Barakat , Field and Taylor, 2013), possibly due to their larger thenar musculature. Whether in extension or flexion forceful precision grips are achieved through high potential torques of human musculature compared to great apes (Marzke et al., 1999).During finger opposition, large human thenar muscles allow a forceful compound movement of axial rotation, flexion and abduction of the human thumb (Napier, 1961; Halilaj et al., 2014; Feix, Romero, Schmiedmayer, Dollar \& Kragic, 2016; D’Agostino, Dourthe, Kerkhof, Stockmans \& Vereecke, 2017). The larger and flatter sellar-facet (Tocheri, Razdan, Williams \& Marzke 2005; Marzke et al., 2010), as well as a less curved proximal Mc1 and a shorter palmar beak (Niewoehner, 
2005; Marchi, Proctor, Huston, Nicholas \&Fischer, 2017), are associated with greater TMc joint mobility in humans compared to other great apes (Cooney, Lucca, Chao \& Linscheid, 1981; Rose, 1992). Although the high radio-ulnar congruence at the TMc joint may limit abduction, it facilitates resistance to large axial forces generated in human manipulation (Marzke et al., 2010; 2013).

\section{Pongo}

Orangutans are primarily arboreal and engage in quadrumanous torso-orthograde locomotion (Thorpe \& Crompton, 2006; Manduell, Morrogh-Bernard, \& Thorpe, 2011). Hand use during arboreal locomotion is not well studied (Thorpe \& Crompton, 2005), but orangutans are thought to habitually use hook-grips or power-grips that only recruit their fingers (Sarimento, 1988; Rose, 1988). However, orangutans may oppose the thumb to the fingers when climbing small-diameter substrates (Sarmiento, 1988), and preliminary behavioural evidence shows more frequent recruitment of the thumb than traditionally thought (McClure, Phillips, Vogel \& Tocheri, 2012).

In captivity orangutans do recruit the thumb in pad-to-side precision grips during manipulative tasks (Christel, 1993; Bardo et al., 2017). However, they far more frequently use a power-grip, especially for larger objects (Pouydebat, Gorce, Coppens \& Bels, 2009), or a 'V-pocket' grip (Marzke, et al., 2015), in which the object is held in the webbing between the full thumb and index finger (Bardo et al., 2017). In both grips the orangutan thumb may provide support but is unlikely to be strongly recruited, as its relative length would make articulation of the first distal phalanx with all but the largest objects in a power hook grip challenging. Further, this distal phalanx is difficult to articulate with an object already held by the V-pocket at the base of this digit. Orangutans frequently reposition tools with their mouths rather than with their hand (Christel, 1993; Bardo et al., 2017). In the wild, orangutans have not yet been observed using precision grips, even during tool production and use of tools (e.g. van Schaik, Fox \& Sitompul, 1996; Fox, Sitompul, \& van Schaik 1999).

The lack of thumb recruitment in orangutan grips is likely because the orangutan thumb is the shortest, relative to the fingers, of any great ape (Tuttle, 1969; Almécija et al., 2015; Bardo et al. 2018). As such, the theoretical 'work space' for manipulating small objects between the tip of the thumb and the tip of the index finger, a' tip-to-tip' grip, has been shown to be the smallest of all great apes (Feix, et al., 2015). The manipulative capability of orangutans is also constrained by a lack of a distinct flexor pollicis longus that inserts on the distal phalanx, as well as the well-developed thenar musculature, found in humans (Strauss, 1942; Tuttle, 1969; Zihlman, Mcfarland \& Underwood, 2011). Orangutans, however, have the largest range of hyperextension $\left(25^{\circ}\right)$ and radioulnar movement at the first McP joint $\left(36^{\circ}\right)$ of all non-human great apes, especially ulnarly (Tuttle, 1969). This range of movement may relate to the fact that unlike other non-human great apes, the palmar aspect of the orangutan Mc1 head is rotated ulnarly relative to its base, which is argued to be a consequence of the short thumb opposing the rigid palm rather than mobile fingers in this species (Drapeau, 2005). This McP joint mobility may partially offset a TMc joint that has been described as generally more congruent in orangutans than in other great apes, including humans, which presumably limits its range of motion somewhat (Rafferty, 1990). However, the range of movement at this joint has also been described as highly variable (Rafferty, 1990) and a quantitative study found few significant differences in surface congruity at this joint between orangutans and other great apes (Marzke et al. 2010). 


\section{Pan troglodytes}

Chimpanzees predominantly knuckle-walk, a mode of locomotion that does not recruit the thumb (Doran, 1996; Wunderlich \& Jungers, 2009). However, chimpanzees are also arboreal and those of the Taï Forest frequently vertically climb or scramble in trees (Doran, 1993), and this species has been described as more arboreal than gorillas (Remis, 1995; Doran, 1996; Thorpe \& Crompton, 2006). Depending on branch diameter, chimpanzees use their thumbs in adducted, abducted, and opposed positions during power or hook grips (Hunt, 1991; Marzke \& Wullstein 1996; Neufuss Robbins, Baeumer, Humle \& Kivell 2017). Unlike gorillas, chimpanzees only oppose the thumb in-line with, rather than wrapping it around, arboreal substrates during diagonal power grasping (Marzke et al., 1992; Alexander, 1994; Neufuss et al., 2017).

The chimpanzee thumb is frequently involved in tip-to-tip and pad-to-side precision grips during manipulative activities in captivity (Christel, 1993; Marzke \& Wullstein, 1996; Jones-Engels \& Bard, 1996; Pouydebat, Reghem, Borel \& Gorce, 2011). In the wild, rare pad-to-pad precision grips have been observed in Mahale chimpanzees during feeding but pad-to-side grips are the most frequent, employing an adducted thumb (Marzke et al, 2015). This study also highlighted that the pad-to-side grip was used with considerable force when pulling against slender resistant materials such as small diameter branches, vines, grasses and meat fibres (Marzke et al., 2015).

These observed grips may be a result of the chimpanzee thumb to finger ratio that is intermediate between gorillas and orangutans (Drapeau \& Ward 2007; Almécija et al., 2015). Chimpanzees generally have smaller thenar muscles than those of humans (Ogihara, Kunai \& Nakatsukasa, 2005) that can generate lower potential torques, due to shorter moment arms (Marzke et al., 1999). Conversely, the transverse head of the adductor pollicis muscle is equivalent to or larger in chimpanzees than in humans and can create larger potential torques (Tuttle, 1969; Marzke et al., 1999; Jacofsky, 2009). As flexor pollicis brevis and opponens pollicis muscles tend to secondarily adduct the TMc joint in chimpanzees, while they abduct the joint in humans, Marzke et al., (1999) have linked this myological morphology to adduction of the thumb in pad-to-side grips in this species. The chimpanzee TMc joint itself is relatively incongruent, especially dorso-palmarly, which may allow for mobility at this joint at the cost of stability (Rafferty, 1990; Marzke et al., 2010).

\section{Pan paniscus}

Like chimpanzees, bonobos also primarily knuckle-walk, both arboreally and terrestrially, which does not recruit the thumb. However, they are argued to be more arboreal than chimpanzees (Alison \& Badrian, 1977; Susman \& Badrian, 1980; Crompton, Sellers \& Thorpe, 2010) and engage in arboreal palmigrady more frequently (Doran, 1993). While the thumb is frequently observed in use during vertical climbing and suspension, it may not be meaningfully loaded (Samuel et al., 2018). While the thumb may be recruited in palmigrady, data on this are lacking and so it is possible the thumb is most frequently loaded during manipulative behaviour in this species.

Captive bonobos use precision grips and, uniquely among non-human great apes, they can independently flex their first distal phalanx while performing them (Christel, 1993; Christel, Kitzel \& Niemitz, 1998). The most frequent grips used by bonobos during manipulative tasks, that employ the thumb, are the V-pocket and pad-to-side grips, in which the thumb is adducted (Bardo, Borel, Meunier, Guéry \& Pouydebat, 2016). In naturalistic environments bonobos use, albeit rarely, tools in social behaviours and to shelter from rain (Ingmanson, 1996; Hohmann \& Fruth 2003; Furuichi et al., 
2015). Sanctuary-living bonobos have also been reported to employ a variety of different grips during nut-cracking, including many that involve an adducted thumb that may be flexed or extended (Neufuss, Humle, Cremaschi \& Kivell, 2016).

Bonobos have a similar relative thumb length (Almécija et al., 2015) and a comparable kinematic workspace (Feix et al., 2015) to chimpanzees. This species has well-developed thenar musculature that can exert high pressures at the TMc joint and includes a tendon of the flexor digitorum profundus that flexes the distal phalanx (van Leeuwen et al., 2018), a trait that is weakly expressed or absent in chimpanzees (Tuttle, 1969; Susman, 1998). However, the bonobo thumb is not capable of the same level of force as is the human thumb (van Leeuwen et al., 2018). Further this species demonstrates a fusion of thumb and index finger musculature that may limit complex precision grips that require independent movement of these digits (van Leeuwen et al., 2018). The shape of the bonobo TMc joint is similar to that of humans however unlike humans strong volar ligaments at this joint in bonobos restrict the extension at the TMc to just $30^{\circ}$ (van Leeuwen et al., 2018b). The rounded Mc1 base palmar beak is also thought to limit axial rotation and medio-lateral movements of the Mc1 on the trapezium and therefore the compound movement involved in pad-to-pad opposition grips (van Leeuwen et al., 2018b).

\section{Gorilla}

The most frequent locomotor mode in gorillas is terrestrial knuckle-walking, which does not recruit the thumb (Inouye, 1994; Remis, 1998; Matarazzo, 2013). Gorillas are also arboreal and when captive lowland gorillas climb large diameter supports, they recruit, but do not oppose, the thumb keeping it in line with the rest of the digits (Sarmiento, 1994). Unfortunately, relatively little is known about wild western lowland gorilla hand use compared to that of mountain gorillas (Byrne, Corp \& Byrne,2001; Neufuss et al., 2017). Wild mountain gorillas also adduct their thumbs in grips of $>50 \mathrm{~cm}$ diameter substrates but they oppose the thumb in-line with, or around, $6-10 \mathrm{~cm}$ diameter substrates and the thumb is particularly important in counter-stabilising descent grips on lianas (Neufuss et al., 2017). While arboreal behaviours may have been traditionally underestimated in gorillas (Crompton et al., 2010; Neufuss et al., 2017), this genus most frequently terrestrially knuckle-walks, which does not recruit the thumb, and therefore the thumb may be most often used during manipulation.

Captive gorillas can perform 'tip-to-tip' precision grips (Christel, 1993; Pouydebat, Laurin, Gorce \& Bels, 2008), although they also often use power grips and 'interdigital brace' grips during manipulative tasks (Bardo et al., 2017). The latter grip threads an object between the adducted thumb and index finger as well as the palmar or dorsal aspects of the ulnar digits (Lesnik, Sanz \& Morgan et al., 2015; Bardo et al., 2017). Wild mountain gorillas most frequently employ precision grips that adduct the thumb during food processing, including interdigital brace, thumb wrap, and pad-to-side grips (Neufuss, Robbins, Baeume, Hulme \& Kivell 2018). The latter grip is reported as the most frequent and the authors suggest this may due to the fact that gorilla thumb may not be able to resist the seemingly forceful grips observed, in tip-to-tip position (Neufuss et al., 2018). However, Neufuss et al. (2018) have emphasized the great variety of grips and thumb positions used by mountain gorillas in food processing and while they did not observe precise in-hand manipulation (sensu Landsmeer, 1962) in this community, it has been reported in others (Byrne et al., 2001; Bardo 
et al., 2017). Gorillas have also been observed engaging in tool-use behaviours (Breuer, NdoundouHockemba \& Fishlock, 2005; Kinani \& Zimmerman, 2015).

The use of the gorilla thumb in a variety of grips may be linked to its long thumb, relative to the fingers, which is relatively longer than that of all non-human great apes (Susman, 1979; Almécija et al., 2015). These hand proportions provide for the largest theoretical kinematic workspace, between the thumb and index finger, compared with all other non-human great apes (Feix et al. 2015). The Mc1 head is of comparable breadth to that of humans, which may facilitate a similar degree of movement at the McP joint in gorillas (Hamrick \& Inouye 1995; Susman, 1998). The distal fibres of abductor pollicis longus muscle do not separate into a distinct muscle belly in gorillas (contra Sarminento, 1994), which is the extensor pollicis brevis muscle in humans. However, the abductor pollicis longus muscle does insert on the proximal phalanx more frequently than in other great apes (Diogo et al., 2012), which may facilitate increased thumb dexterity. Similarly, gorillas have a less congruent TMc joint than Pongo (Rafferty, 1990) allowing for a greater range of motion at this joint, although this difference is small quantitatively (Marzke et al., 2010) and different approaches used to quantify TMc joint surface congruence are often difficult to compare (Halilaj et al., 2014b).

\section{Predictions}

All non-human great apes appear to habitually use pad-to-side or V-pocket grips in which the thumb is adducted, and variably flexed (Jacofsky, 2009; Marzke et al., 2015; Bardo et al., 2016; 2017; Neufuss et al., 2018). While detailed quantitative data on the level of in vivo flexion during these grips in the species is sparse, the pad-to-side grip typically involves contacting the side of the index finger with the distal thumb pad in non-human great apes, a movement that intuitively requires little flexion of the first McP (Fig. 1b top), relative to pad-to-pad opposition with the fifth distal phalanx, for example. Conversely, the V-pocket griprequires even less flexion of the McP to maintain a grip on an object between the ulnar aspect of the first proximal phalanx and the radial side of the second metacarpal. Thus while the first McP can flex further than it can adduct in non-human great apes, the full range of flexion does not appear to be used in these habitual grips. Therefore, we predict that both subarticular relative trabecular volume (RBV/TV, see methods) and degree of anisotropy (DA) will be greatest disto-palmarly in the ulnar aspect of Mc1 head of these species, reflecting moderate flexion and adduction of the first McP (H1a; Fig. 1b). Gorillas may be an exception to this pattern because while they frequently adduct the thumb in interdigital brace grips (Bardo et al., 2017), they also have a wide Mc1 head (Hamrick \& Inouye 1995; Susman, 1998) and frequently recruit the thumb in abducted positions (Neufuss et al., 2018). As a result we expect that gorillas may have lower DA across the Mc1 head than other non-human great apes (H1b). Conversely, even though the human thumb is also recruited in many different postures, due to frequent use of forceful precision grips in which the thumb is flexed and abducted (Napier 1956; Feix et al., 2016; Marzke, 2013), we predict that humans will have greater subarticular DA and RBV/TV in the radiopalmar aspect of the Mc1 head relative to all other great apes studied (H1c; Fig. 1a).

Given gorillas have been shown to use a variety of thumb positions during manipulation we predict they will demonstrate lower DA throughout the Mc1 base than other non-human great apes, which themselves, will have a relatively uniform distribution of DA values across the TMc joint due to less frequent thumb use than in humans (H2a). We also predict RBV/TV values will be greatest in the palmar aspect of the Mc1 base in great apes (H2b; Fig. 1b) as the TMc joint is primarily flexed in 
these species, during both arboreal power grasping (Neufuss et al., 2017) and precision grasping (Marzke, 1997; Marzke et al., 2015; Bardo et al., 2016; 2017; Neufuss et al., 2018). While manipulative grips habitually practised by non-human great apes involve adduction of the thumb, this movement may be restricted at the TMc joint by a thicker anterior oblique ligament in nonhuman great apes compared with that of humans (van Leeuwen et al., 2018b). The rounded palmar beak at the Mc1 base in non-human great apes, relative to humans, is also thought to restrict axial rotation of the Mc1, and thus adduction (Marzke et al., 1992; van Leeuwen et al., 2018b). When similar pad-to-side grips are experimentally performed in humans, the contact area of the TMC surfaces is larger ulnarly but the closest point between them is at the palmar beak of the Mc1 (D'Agostino et al., 2017; Fig. 1b). Therefore, we predict flexion will be the dominant habitual movement in these grips, rather than adduction, reflected by the trabecular distribution in the nonhuman primate TMc joint (Fig. 1b, bottom). In humans we predict RBV/TV and DA will be greater in the radio-palmar aspect of the Mc1 base (H2c; Fig.1a), due to habitual abduction and flexion during precision grasping (Napier, 1956; Feix et al., 2016; D'Agostino et al., 2017) as has been demonstrated before for RBV/TV (Stephens et al., 2018).

\section{Materials}

Subarticular trabecular bone was analysed in the Mc1 of Homo sapiens $(n=10)$, Pan paniscus $(n=10)$, Pan troglodytes ( $n=11)$, Gorilla gorilla gorilla $(n=10)$, Pongo sp. $(n=1)$, Pongo pygmaeus $(n=5)$ and Pongo abelii ( $n=3$, Table 1 ). All specimens were considered adult based on complete epiphyseal fusion of the Mc1 and other postcranial elements, free from external signs of pathology and all nonhuman specimens were wild-shot. Human specimens were drawn from four populations: Nubians of 5th century AD Sayala, Egypt (Strouhal \& Jungwirth, 1979; Paoli et al., 1993), Yámanas individuals from 19th century Tierra del Fuego (Marangoni et al., 2011), 20th century individuals from Syracuse, Italy and 20th century individuals from a cemetery in Inden, Germany (Grosskopf, 2015). The samples were sex balanced for each species, although one Pongo pygmaeus and two human specimens were of unknown sex. For the non-human apes an effort was made to analyse even numbers of left and right Mc1s, as there are some signs of lateral asymmetry in metacarpal trabecular (Stephens et al., 2016) and cortical bone (Sarringhaus, Stock, Marchant \& McGrew, 2005). However, these differences are slight in absolute terms (Sarringhaus et al., 2005; Skinner et al., 2015) and so Mc1 side unlikely to meaningfully affect the current analysis. Conversely humans are cross-culturally right-handed (Faurie et al., 2005) and this is reflected in Mc1 trabecular bone (Stephens et al., 2016; Reina et al., 2017). Therefore the human sample was drawn from right hands to avoid potential bias related to handedness.

\section{Methods}

\section{MicroCT Scanning}

Specimens were scanned with a BIR ACTIS 225/300, Diondo D3, or a Skyscan 1172 high resolution microCT scanner at the Department of Human Evolution, Max Planck Institute for Evolutionary Anthropology, Germany, or with the Nikon 225/XTH scanner at the Cambridge Biotomography Centre, University of Cambridge, UK. Scans were performed at 100-160kV and 100-140 $\mu \mathrm{A}$, using a brass or copper filter of $0.25-0.5 \mathrm{~mm}$. The scans were reconstructed to create images with an isometric voxel size of $28-41 \mu \mathrm{m}$ depending on the size of the specimen. 


\section{Image processing}

Avizo 6.3 (Visualization Sciences Group) was used to isolate and rotate micro-CT scans of each Mc1 into a standardised anatomical position (Fig. 2a) and the Ray Casting Algorithm (Scherf \& Tilgner, 2009) was used to segment bone tissue. Trabecular structure was analysed with the whole-epiphysis method, which has been described and tested in detail (Gross, Kivell, Skinner, Nguyen \& Pahr, 2014). Briefly, medtool 4.2 (Dr. Pahr Ingenieurs e.U.) was used to run the image through a series of image filters that separated the inner trabecular structure from the cortical shell (Fig. 2b). Specifically, an algorithm casts mathematical rays from the edge of the cortical bone inward in seven directions, the three orthogonal axes as well at the four diagonals of the unit cube. Where at least five of these seven rays met the first 'inner-air' voxel, that is part of the image that is not bone and inside the cortical shell, they were marked as part of the inner structure. A smooth kernel, with a diameter equal to the measured average trabecular thickness in that bone, was then used to close the gaps in this inner structure, the trabeculae, to delimit the volume of the inner trabecular structure (Pahr \& Zysset, 2009). A three-dimensional (3D) grid was then superimposed on the inner structure and overlapping spherical volumes of interest (VOI) with a $5 \mathrm{~mm}$ diameter were positioned at each vertex within the $2.5 \mathrm{~mm}$-spaced grid. Trabecular bone volume (BV/TV) and degree of anisotropy (DA) were then measured for each VOI (Fig. 2c) as several studies have demonstrated these properties correlate with bone biomechanics (Odgaard, 1997; Uchiyama et al., 1999; Pontzer et al., 2006; Barak et al., 2011; Lambers et al., 2013), and are not strongly affected by allometry (Doube, Kłosowski, Wiktorowicz-Conroy, Hutchinson \& Shefelbine 2011; Barak, Lieberman \& Hublin, 2013b; Ryan \& Shaw, 2013). The mean intercept length (MIL) method was used to calculate the second order fabric tensor and DA as 1 - (lowest eigenvalue / greatest eigenvalue). Thus, DA values of 0 represent total isotropy and values of 1 represent total anisotropy. Each trabecular variable was then separately interpolated on 3D tetrahedral mesh created using CGAL (www.cgal.,org ; Fig. 2d). The outer surface of this trabecular mesh was then isolated using Paraview (www.paraview.org), and smoothed to permit landmark sliding (see below) in Meshlab (Cignoni et al., 2008) via a screened Poisson surface reconstruction filter (Kazhdan \& Hoppe, 2013; Fig. 2e). For left Mc1s, this smoothed mesh was oriented in the same way as right Mc1s by a reflection filter in Meshlab to allow for homologous comparisons.

\section{Geometric morphometric mapping}

Only the subarticular trabecular bone of the Mc1 head and base was analysed rather than the entire volumetric trabecular model created by the whole-epiphysis approach. This subarticular trabecular bone is the first point of transmission for external loads from the cortical shell to the deeper trabecular structure and should contain a functional adaptation signal (Marzke et al., 2010; Zhou et al., 2014, Sylvester \& Terhune, 2017). We apply 3D geometric morphometric (GM) techniques (Gunz \& Mitteroecker, 2013) to the analysis of trabecular bone (Dunmore et al.,2019) in a similar manner to the method described by Sylvester \& Terhune (2017).

\section{Anatomical Landmark definitions}

Many landmark sets have been used to analyse the primate Mc1 proximal base (Niewoehner, 2005; Marchi et al., 2017), and recently a set has been used to analyse the shape of the primate Mc1 head (Galletta, et al., 2019) The location and type (Bookstein, 1991) of anatomical landmarks used here for the head and base of Mc1 are given in Tables 2 \& 3, respectively. Previously identified cortical landmarks employed in these studies were accurately transposed to the inner trabecular surface as 
thin cortical bone at the metacarpal head and base in hominids (Tsegai et al., 2017) allows for high correspondence between these surfaces.

\section{Repeatability}

Three random Mc1 specimens from each species were landmarked on their head and base, five times respectively, over several days with Checkpoint (Stratovan Corporation, Davis, CA), following Fernandez et al. (2015). The Morpho package in Rv3.3.0 (Schlager, 2017; R Development Core team, 2016) was then used to generate Procrustes coordinates for the five repeats of three individuals per species and articular surface. These coordinates were then plotted on the first two principle components (PCs) of each of the 10 repeatability comparisons (Supp. Fig1). Pairwise permutational MANOVAs, with Bonferroni correction, conducted on PC1 and PC2 scores demonstrated that repeats of individual configurations were significantly different from the other two specimens in each case, so landmarks were considered repeatable (Supp. Fig1).

\section{Geometric morphometric procedure}

Both landmark templates (Fig. 2f) were created by defining sliding semi-landmarks on curves at the subarticular surface margins of a random specimen in Checkpoint. These curves were each bordered by anatomical landmarks following Gunz, Mitteroecker \& Bookstein (2005). For the Mc1 head template, single sliding semi-landmarks were defined on each of the eight curves. For the Mc1 base template, three sliding semi-landmarks were defined for each of the four curves between anatomical landmarks. Where subarticular margins were smoothed, a translucent model of the cortical surface was overlaid in Paraview to ensure correct placement of the template landmarks. Additional sliding semi-landmarks were then distributed over each subarticular surface in Avizo 6.3 (Visualization Sciences Group, Germany) to produce a 49 landmark template for the Mc1 head, comprising nine anatomical landmarks, eight sliding semi-landmarks on curves and 32 surface sliding semi-landmarks. The 40 landmark template for the base contained five anatomical landmarks, 12 sliding semi-landmarks on curves and 23 surface sliding semi-landmarks (Fig. 2f). Subsequently, anatomical landmarks were placed on every specimen and then each landmark template was projected onto each of the other $49 \mathrm{Mc1}$ heads and 48 bases, respectively, using the Morpho package in $\mathrm{R}$ (Schlager, 2017). A single Pongo pygmaeus specimen did not have a fully-preserved base and was excluded from the base analyses. Each template was relaxed onto the surface of each Mc1 by minimising bending energy and then semi-landmarks were slid along their respective curves or surfaces by minimising Procrustes distances, using the Morpho package in R (Schlager, 2017) .

\section{Data mapping}

A custom Python script was run using Paraview to allow the non-smoothed surface mesh triangles to inherit trabecular values (BV/TV \& DA) from their originating tetrahedra. The Python module SciPy (Jones, Oliphant \& Peterson, 2001) was then used in medtool 4.2 (Dr. Pahr Ingenieurs e.U.) to interpolate the trabecular values to the closest landmark (Fig. 1g). This procedure is analogous to measuring trabecular structure with $1 \mathrm{~mm}$ diameter spherical VOIs, centred $0.5 \mathrm{~mm}$ deep to the normal of the inner trabecular surface, at the location of a landmark. A Procrustes procedure was then performed using the geomorph package (Adams, Collyer, Kaliontzopoulou \& Sherratt, 2017) in $\mathrm{R}$ to produce two sets of homologous landmarks each with the trabecular parameters mapped to them (Fig. 2g). 


\section{Relative trabecular volume (RBV/TV)}

A relative measure of bone volume fraction (RBV/TV) was calculated for interspecific comparisons, in which the raw BV/TV values were divided by the mean of all landmark BV/TV values on that subarticular surface. If a landmark value is close to the average of that Mc1 surface it will have a value of $\sim 1$, whereas a landmark with a lower than the average BV/TV will have an RBV/TV $<1$ and with a higher value will have RBV/TV $>1$. This standardisation of BV/TV values was performed for several reasons. BV/TV can vary systemically across species (Tsegai, Skinner, Pahr, Hublin \& Kivell 2018) and thus may diminish the potential functional signal under investigation here. Further, while $\mathrm{BV} / \mathrm{TV}$ yields functional information relating to the response of trabecular bone to both magnitude and direction of load, it conflates these signals. The present study is focussed on the latter response, as it is more directly related to thumb joint postures in extant hominids. RBV/TV landmark values reflect the distribution of trabecular bone beneath an articular surface, in any given bone, irrespective of the average, or global, subarticular BV/TV present. Where this RBV/TV distribution is uneven, higher landmark values are consistent with habitual loading of articular surface in that region and, in turn, consistent with joint postures that load the surface in this manner. Comparisons of these values between groups therefore test for differences in the distribution of bone volume and thus for differences in habitual loading postures at a joint. If these values were not standardised, the region of highest landmark values on a given subarticular surface may not be differentiated from the lowest values on another subarticular surface with higher average, or global, BV/TV. That is, despite different distributions of BV/TV across these two subarticular surfaces, reflecting different habitually loaded joint postures, these values appear the same. Standardising these BV/TV values is therefore necessary to assess if their subarticular distribution, rather than magnitude, varies between the species studied (Sylvester and Terhune, 2017; Sukhdeo, Parsons, Niu \& Ryan, 2018). Further, intraspecific variation in BV/TV has been shown to be considerable in a large sample of humans, yet the relative differences in BV/TV at several VOIs appear to show a consistent functional signal across populations (Saers, Cazorla-Bak, Shaw, Stock \& Ryan et al., 2016). Therefore, here we opt to use a relative measure to somewhat control for non-functional trabecular signals and analyse which areas of the subarticular trabecular bone have most adapted to habitual loads.

\section{Statistical analysis}

In order to test for significant differences in the distribution of subarticular RBV/TV and DA values between species a dual statistical approach was employed. In order to investigate where subarticular regions were significantly different between species, trabecular values at each landmark were compared using univariate statistics. These regional comparisons, however, do not assess if the distribution of values over an entire subarticular surface are significantly different between species. Small areas of significantly different trabecular structure, while functionally interesting, may not sum to a significantly different distribution of trabecular values over a subarticular surface and so must be interpreted with caution. Therefore, as a corollary, variation in trabecular variable distribution over the subarticular surface was compared between species with multivariate analysis. Together this dual, multivariate and univariate, approach tests if the distribution of trabecular values differ over the entire subarticular surface and in which regions these differences occur.

\section{Univariate regional analysis}

For the univariate analysis, significant species differences in trabecular parameters were independently tested for at each landmark using 'mass-univariate' statistics, following Friston et al. 
(1995). Shapiro-Wilk tests identified significantly non-normal data $(p<0.05)$ for both trabecular parameters at some landmarks. Therefore non-parametric Kruskal-Wallis tests were run at each homologous landmark for consistency. If these omnibus tests were significant at a given landmark post-hoc Dunn's tests (Dunn, 1964) were used to identify significant pairwise species differences at $p<0.05$ after a Bonferroni correction (Dunn, 1961). Trabecular values were compared at homologous landmarks between species rather than with spatially correlated neighbouring landmarks. Polarity and the effect size of pairwise comparisons were determined via Z-scores. Significant univariate species differences at each landmark could then be mapped to an average Mc1 model, to show regional differences for functional interpretation. Though Dunn's test is conservative (Dunn, 1964) this univariate approach may still be subject to Type I error. Therefore significant trabecular value differences were only considered functionally meaningful if they occurred at a minimum of four spatially contiguous landmarks (as this was $8-10 \%$ of each template) to further ameliorate Type I error.

\section{Multivariate whole-surface analysis}

To investigate whether distribution of RBV/TV and DA were different between species over the whole subarticular surfaces multivariate analyses were performed. For Mc1 head and base subarticular surfaces, separately, a principle components analysis (PCA) was performed using variation in RBV/TV or DA at each landmark as a variable, resulting in four PCAs. In each PCA individual Mc1s are represented by a number of principle component (PC) scores. Since three first three principal components cumulatively explain the majority, in this case more than $65 \%$, of the variation, the first three $\mathrm{PC}$ scores of each individual were combined into a multivariate response variable. An omnibus one-way permutational MANOVA was then used to test for significant differences in this multivariate response variable across species. Where this omnibus test was significant, subsequent pair-wise MANOVA's indicated that the distribution of either RBV/TV or DA values was significantly different between these species. These tests were run using the Vegan package (Oksanen et al., 2018) in Rv3.3.0 (R Core Development team, 2016) and the pairwise tests were run with a Bonferroni correction. To visualise inter-specific differences, and intra-specific variation, in subarticular value distribution across Mc1 subarticular surfaces each PCA is plotted. The landmarks at which trabecular values provided the largest negative or positive loadings for a particular PC are visualised on the subarticular surface at the negative and positive end of the PC respectively, similar to the approach of Sylvester and Terhune (2017). This aids in the post-hoc qualitative interpretation of exactly where trabecular value distributions are different between species, but does not specifically test for this as the univariate regional analysis does.

\section{Results}

\section{Average Species values and Univariate landmark comparisons}

Average values at each landmark, per species are depicted in for RBV/TV (Fig. 3a) and DA (Fig. 3b) respectively. Significantly different values at each landmark, in each pairwise species comparison are depicted for RBV/TV (Fig. 4) and DA (Fig. 5) for both subarticular surfaces of the Mc1. As post-hoc Dunn's test pairwise comparisons were too numerous to be easily interpreted in table format, the effects size of each test, the Z-test statistics, are summarized in Table 4. These Z-scores were transformed into unsigned, absolute values to demonstrate the size of differences in trabecular values between species at each subarticular surface. 


\section{Homo sapiens}

$H$. sapiens had the largest range of average RBV/TV values among the sample, with higher values at disto-palmar landmarks on the radial aspect of the Mc1 head (Fig.3a). This region had significantly higher RBV/TV values compared with all other great apes (Fig. 4). In the Mc1 base, average RBV/TV values were highest radio-palmarly, although not at the most palmar landmarks (Fig.3a). H. sapiens had significantly higher RBV/TV at landmarks in the central and radial aspects of the Mc1 base compared to with Gorilla and P. troglodytes (Fig.4). H. sapiens displayed the highest average DA values throughout the head compared to all other species (Fig.3b), which resulted in significantly higher values than Gorilla at almost every landmark (Fig.5). Further, H. sapiens had significantly higher DA than $P$. troglodytes, as well as Pongo, at disto-ulnar landmarks and $P$. paniscus at palmar landmarks. The average $H$. sapiens DA values were highest in the radio-palmar and ulnar aspects of the Mc1 base. $H$. sapiens Mc1 base landmarks had significantly higher DA values than $P$. paniscus in the radio-palmar region and than Gorilla in the central palmar region.

\section{Pongo}

In the Mc1 head, the average RBV/TV in Pongo was highest in the ulno-distal region (Fig.3a). Pongo had significantly greater RBV/TV at landmarks situated ulno-dorsally than Gorilla and H. sapiens (Fig.4). In the average Mc1 base, Pongo displayed a slightly higher RBV/TV in the central palmar region, though the range of values throughout the base was small (Fig.3a). Pongo did not have a region of significantly different RBV/TV from other species in the Mc1 base. DA in the Pongo average Mc1 head was lowest ulno-distally (Fig.3b) and significantly higher than Gorilla in the palmar region (Fig.5). In the base, Pongo had a slightly higher average DA ulnarly but did not show a contiguous patch of landmarks significantly different from any other species, expect $P$. troglodytes where Pongo had significantly higher DA at radio-central landmarks.

\section{Pan troglodytes}

P. troglodytes had the highest RBV/TV values at ulnar landmarks on the disto-palmar aspect of the Mc1 head (Fig. 3a). RBV/TV in this species was significantly higher at radio-palmar landmarks compared to Pongo and at ulno-palmar landmarks relative to $H$. sapiens (Fig. 4). The Mc1 base in $P$. troglodytes had the highest range of average RBV/TV values among non-human great apeMc1 bases (Fig. 3a). Average RBV/TV was highest in the central palmar base but values were only significantly greater than $\mathrm{H}$. sapiens, at dorso-ulnar landmarks. The Mc1 head of $P$. troglodytes had lower DA at disto-palmar landmarks on its ulnar side (Fig. 3b). P. troglodytes DA was only significantly greater than Gorilla, across radial and dorsal landmarks (Fig. 5). The highest average DA values were in the dorsal Mc1 base of $P$. troglodytes and these DA values were significantly higher than Gorilla, $H$. sapiens and, to a lesser extent, Pongo at radio-dorsal landmarks. DA values were also significantly higher than P. paniscus in this species at radio-palmar Mc1 base landmarks (Fig. 5)

\section{Pan paniscus}

$P$. paniscus possessed the lowest range of average RBV/TV values in the Mc1 head across the sample. The homogenous distribution of RBV/TV values in this species resulted in significantly higher RBV/TV than in Gorilla at dorso-ulnar landmarks and H. sapiens at both dorso-ulnar as well as palmoulnar landmarks (Fig. 4). In the Mc1 base, P. paniscus had slightly higher average RBV/TV values in its central palmar landmarks, although like Pongo the range of values was low throughout the base. This species had significantly higher RBV/TV relative to $H$. sapiens at the most extreme dorsally- 
positioned landmarks in the Mc1 base. For DA, the $P$. paniscus Mc1 head had a similar average pattern to $P$. troglodytes, although lower average DA values were found at more palmar landmarks (Fig. 3b). DA values of the Mc1 head were significantly higher than in Gorilla at dorsal and radial landmarks (Fig. 5). In the average Mc1 base, P. paniscus showed higher DA values at dorsal landmarks (Fig. 3b) that were significantly greater, especially radially, than in $\mathrm{H}$. sapiens and Gorilla (Fig. 5).

\section{Gorilla}

Gorilla had the highest range of average RBV/TV values across Mc1 head landmarks in non-human great apes. The highest RBV/TV values were located ulnarly on the disto-palmar aspect of the Mc1 head (Fig. 3a). Gorilla was significantly higher in RBV/TV than H. sapiens ulno-palmarly and significantly higher than all other great apes disto-palmarly (Fig. 4). The average Gorilla Mc1 base had higher RBV/TV values centred at its most palmar extent. RBV/TV was only significantly higher than that of $H$. sapiens, at dorso-ulnar landmarks (Fig. 4). Gorilla had the lowest average DA of all species throughout both the Mc1 head and base subarticular surfaces (Fig. 3b). This species showed significantly lower DA than $\mathrm{H}$. sapiens at most landmarks on the Mc1 head as well as at radial and dorsal landmarks in comparison with P. troglodytes (Fig. 5). Relative to P. paniscus, Gorilla again had significantly lower DA at dorsal and radial landmarks, but these were more distally and centrally located than in the comparison with $P$. troglodytes. Pongo had significantly higher DA relative to Gorilla at centrally located disto-palmar Mc1 head landmarks (Fig.5). Gorilla had low average DA throughout the Mc1 base and significantly lower DA than both Pan species at central and more dorsally located landmarks (Fig.5).

\section{Multivariate whole-surface comparisons}

PCA results for RBV/TV and DA value distributions in the Mc1 head and base are depicted in Figures 6 and 7 respectively. Permutational MANOVAs were run using the first three PCs of each subarticular surface, as further PCs each explained less than $10 \%$ of the variance in each PCA. These omnibus tests were significant for both RBV/TV and DA for in the head and base (Table 5) indicating there were significant differences between species in overall subarticular trabecular value distribution. The pairwise permutational MANOVA results generally matched those of the univariate comparisons, that is significant subarticular surface value distribution differences were found between species with significantly different trabecular values at numerous subarticular landmarks.

\section{Mc1 heads}

The first principal component (PC1) of the RBV/TV data explained $36 \%$ of the variation and reflected variation in RBV/TV values at dorsal and palmar Mc1 head landmarks. PC2 represented $27 \%$ of the variation in RBV/TV values at radio-palmar and disto-ulnar landmarks, whereas PC3 explained $14 \%$ of the variation and reflected radio-ulnar landmark variation (Fig. 6a). Pairwise permutational MANOVAS demonstrated that $H$. sapiens was significantly different in RBV/TV distribution compared with all other hominids (Table 5). H. sapiens appear to be distinguished from other species studied primarily by radio-palmarly higher RBV/TV in the Mc1 head, on PC2 and PC3 (Fig. 6a ). Gorilla was also significantly different from both Pan species (Table 5) with apparently higher RBV/TV values ulno-palmarly (Fig. 6a). 
For DA, PC1 described $53 \%$ of the variation and reflected lower or higher values at most landmarks across the Mc1 head (Fig. 6b). PC2 explained $12 \%$ of the variation and reflected variation in DA values at disto-palmar and dorsal landmarks. Pairwise tests revealed that Gorilla was significantly different from all other species (Table 5) likely due its overall lower DA (Figs. 3 and 6b). Conversely $\mathrm{H}$. sapiens was not significantly different in its subarticular DA distribution relative to any other species, except Gorilla, despite being seemingly distinguished by higher DA values at most landmarks on PC1 ( Figs. 3 and 6b). Neither PC2 nor PC3, which only explained 7.3\% of the variation in DA, differentiated the studied taxa to a notable extent.

\section{Mc1 bases}

In the Mc1 base RBV/TV values, 35\% of the variation was explained by values at radio-palmar and a combination of extremely palmar and dorsal landmarks in PC1 (Fig. 7a). PC2 explained $17 \%$ of the variation in RBV/TV values and was driven by radio-ulnar landmark RBV/TV values, while PC3 explained $14 \%$ of the variation and was driven by dorso-palmar landmark values. H. sapiens was significantly different to all other species (Table 5) and appeared to be distinguished by higher RBV/TV in the palmar and radial aspects of the Mc1 base on PC1 (Fig. 7a). Gorilla tended to separate from the other taxa displaying higher RBV/TV at extremely palmar and dorsal landmarks and plotting at the opposite end of PC1, though this species displayed a large range of DA distributions (Fig. 7a), while all other taxa were intermediate between $\mathrm{H}$. sapiens and Gorilla. However, there were no significant differences in RBV/TV of the Mc1 base across non-human great apes (Table 5). While PC2 and PC3 were driven by contiguous patches of landmark values they did not distinguish any taxa (Fig. 7a).

In subarticular DA of the Mc1 base, PC1 explained 38\% of the variation and mainly differences in central and dorsal landmark DA (Fig. 7b). PC2 explained $21 \%$ of the variation reflecting radio-palmar and dorsal landmark DA values. PC3 explained $10 \%$ of the variation and was driven by DA values at radio-ulnar landmarks. The distribution of Mc1 base DA in both $P$. troglodytes and $P$. paniscus was significantly different from $\mathrm{H}$. sapiens and Gorilla (Table 5). This different distribution appeared to be driven by PC2 (Fig. 7b). Both Pan species had highest DA at dorsal landmarks, while the latter two species had their highest DA values at radio-palmar landmarks (Figs.2 and 7b). Higher DA values extended more palmarly in $P$. troglodytes relative to $P$. paniscus (Figs. 2 and $7 \mathrm{~b}$ ) which probably explains their significantly different overall DA distributions (Table 5.) Pongo Mc1 base DA distribution appeared to be intermediate between all species studied on PCs 1 and 2 (Fig. 7b) and was not significantly different from any other species (Table 5).

\section{Discussion}

We investigated variation in the subarticular trabecular bone structure of the Mc1 across extant hominids to test whether it is consistent with what is known about their habitual thumb postures. In the Mc1 head, we predicted that subarticular RBV/TV and DA would be greatest at ulnar distopalmar landmarks in non-human hominids (H1a), with the exception of gorillas that were predicted to have lower DA throughout the head (H1b). Conversely in humans we predicted DA and RBV/TV would be highest at radio-palmar landmarks on the Mc1 head (H1c). We also predicted that gorillas would have the lowest DA within the non-human hominids, which would have relatively uniform DA values across the Mc1 base (H2a). Further, non-human hominids were predicted to have the highest 
RBV/TV values in the central palmar aspect of the Mc1 base (H2b) while humans would have highest $\mathrm{RBV} / \mathrm{TV}$ and DA values radio-palmarly $(\mathbf{H} \mathbf{2 c})$.

\section{Mc1 heads}

The data support our predictions concerning subarticular RBV/TV across hominid Mc1 heads (H1a \& c) but only partially support those relating to DA (H1 b \& c). Average RBV/TV was higher at ulnodistal landmarks in the Mc1 head across all non-human great apes, consistent with a habitually adducted thumb used in pad-to-side or V-pocket grips (Marzke et al., 2015; Bardo et al., 2016; 2017; Neufuss et al., 2016, 2018) and some power grasps used in African ape locomotion (H1a; Neufuss et al., 2017, Samuels et al., 2018). Contrary to our hypothesis, however, DA was lowest at the ulnodistal landmarks, where RBV/TV was highest, in chimpanzees, bonobos and orangutans (H1a). This prediction was based on the concept that stereotypical loading of the first McP joint would cause the realignment of trabeculae, via remodelling, in the direction of this load, resulting in higher DA. Interpreting the results in this way would imply orangutans do not load their Mc1 head in a flexed McP joint posture, the disto-palmar Mc1 articular surface, which does not agree with overwhelming behavioural and anatomical evidence nor the present RBV/TV results. This coincidence of highest RBV/TV and lowest DA values at ulno-distal landmarks instead reflects more trabecular bone with less alignment in this subarticular region that may be better able to withstand load from multiple directions. Indeed, while RBV/TV may be the result of thicker or more trabeculae, or some combination of both, it is notable that this average lower ulno-distal DA pattern was present in the three species with the smallest Mc1s (i.e., P. troglodytes, P. paniscus and Pongo sp.; Fig. 3b) and smaller bones tend to have thicker and fewer trabeculae (Barak et al., 2013b). This DA pattern, therefore, is likely the result of the number of trabeculae and is consistent with high ulnar loading of the McP joint in the chimpanzee, bonobo and orangutan Mc1 head, despite displaying the opposite trend to that predicted. However, further work that accounts for variation in trabecular number is needed to substantiate this interpretation of the present DA results.

For both DA and RBV/TV, the current sample consisting predominantly of Taï chimpanzees ( $P$. troglodytes verus), known to use tools, displayed almost no significant differences from bonobos or orangutans, though the former have been observed using very few tools in the wild (Kano, 1982; Koops, Furuichi and Hashimoto, 2015), and neither are known to engage in percussive tool use (van Schaik et al., 1996; Meulman and van Schaik, 2013). It may be that nut-cracking (Boesch \& Boesch, 1993) and the use of precision forceful grips during food processing (Marzke et al., 2015) are simply not frequent or forceful enough to stimulate subarticular trabecular remodelling in the Mc1 head. A similar sample of Taï chimpanzee Mc1s have previously been shown to have less robust trabecular architecture than another group of chimpanzees that do not habitually nut-crack (Lazenby et al., 2011). Therefore, either a strong osteogenic signal does not exist for this behaviour or it is constrained by more habitual or greater loading of the Mc1 during Taï chimpanzee locomotion (Lazenby et al., 2011; Neufuss et al., 2017).

Gorillas had the highest average RBV/TV at ulnar landmarks situated on the disto-palmar aspect of Mc1 head, consistent with pad-to-side, interdigital brace and 'thumb wrap' grips frequently used in wild manipulation (Neufuss et al., 2018). This subarticular pattern, however, is statistically distinguished from bonobos, orangutans and, to a lesser extent, chimpanzees by the greater range of RBV/TV values across the gorilla Mc1 head, suggestive of more habitual or greater loading of the pollical McP joint in adduction in this species. DA values in the gorilla Mc1 were significantly lower 
than all other species studied and displayed a low range throughout the head (H1b). Combined, this trabecular pattern is consistent with the habitual use of varied thumb positions in gorillas, but also more frequent or forceful loading in thumb adduction, relative to the other non-human great apes (Neufuss et al., 2018). However, while recent work has found that Mc1 head is similarly shaped in both subspecies of gorilla (Galletta, et al., 2019), it must be noted that this prediction was based on the greater volume of behavioural evidence from mountain gorillas (Gorilla gorilla berengei) rather than the western lowland (Gorilla gorilla gorilla) subspecies studied here. Furthermore, there are limited detailed studies of grip and hand use in other non-human hominids in the wild (Marzke et al., 2015) and thus this interpretation must be treated with some caution.

Humans displayed both significantly higher RBV/TV at radial landmarks and higher DA throughout the subarticular Mc1 head than in other great apes $(\mathbf{H 1 C})$. A radio-palmar concentration has also been found in a similar sample of humans, using a method that analysed absolute BV/TV in the whole distal epiphysis of the Mc1 rather than just the subarticular region (Stephens et al., 2018). Further a significantly larger radial palmar epicondyle in the human Mc1 head, relative to other great apes, may also indicate the importance of this region of the Mc1 head to human manipulation (Galletta et al., 2019). This RBV/TV distribution is consistent with a habitually and forcefully opposed thumb, a movement which entails flexion, and importantly, abduction at the McP joint (Napier, 1956). An opposed thumb is used in forceful precision grips during the production (Markze, 1997; 1998) and use of stone tools (Rolian et al, 2011; Key et al. 2018) among other manipulative activities (Napier, 1993; Bardo et al., 2017).

In contrast to these RBV/TV results, high DA throughout the human Mc1 head, while not overall significantly different from any species except gorillas, does not match our prediction of the highest DA at radio-palmar landmarks (H1c). Stephens et al. (2018) found slightly lower average DA values in a similar human Mc1 sample, likely due to their sampling of the whole distal epiphysis, but also found little regional differentiation across the Mc1 head in agreement with the results here. The present result may reflect the higher frequency of forceful prehensile thumb use in humans than in other extant hominids. Though the highest or most habitual forces may be resisted by the radiopalmar McP joint during thumb abduction, the frequently-used, powerful and mobile human thumb likely engenders a stronger osteogenic signal throughout the head than in other apes and therefore more aligned trabeculae (i.e. higher DA). For example, humans are unique among extant hominids in their ability to forcefully extend and stabilise the first proximal phalanx on the Mc1 while flexing the distal phalanx via distinct flexor pollics longus and extensor pollicis brevis muscles (Marzke et al., 1998; Hamrick et al., 1998; Diogo et al., 2012). The thumb held in this position probably induces considerable loads on the distal McP joint, even if these are not as large as those loads resisted in forceful abduction (Cooney and Chao, 1977; Toft and Berme, 1980). The fact that humans appear to be more restricted in McP joint flexion than in other great apes (Tuttle, 1969; Barakat et al., 2013) also supports this interpretation of the human McP joint as a joint restricted by large thenar musculature that can resist higher loads. The insertion of extensor pollicis brevis on the first proximal phalanx (Diogo et al., 2012) may also explain why this high DA was found throughout the subarticular the Mc1 head but not the Mc1 base in humans.

\section{Mc1 bases}

The subarticular trabeculae of the Mc1 base did support our predictions for RBV/TV but not entirely for DA. In concordance with our predictions, average DA throughout the Mc1 base of gorillas was 
lower than other species studied (Fig. 3b) but only significantly different from chimpanzees and bonobos, as these species had significantly higher DA at more dorsal landmarks. Contrary to our predictions, the other non-human great apes did not have a uniform distribution of DA values in the Mc1 base. (H2a). Rather our results indicate a regional pattern, in which chimpanzees and bonobos had the highest DA values in the dorsal Mc1 base and were both significantly different from gorillas. The functional significance of this pattern is not immediately apparent though it may relate to more varied habitual thumb positions in gorillas relative to the other non-human great apes. All nonhuman hominids displayed the highest RBV/TV at extremely palmar and central landmarks consistent with habitual flexion at the TMc joint $(\mathbf{H} \mathbf{2} \mathbf{b})$, though the range of values throughout the base was lower than in humans. This more homogenous distribution of subarticular RBV/TV is consistent with previous studies of absolute BV/TV throughout a similar sample of Pan Mc1 proximal epiphyses (Skinner et al., 2015; Stephens et al., 2016).

In partial support of our predictions humans had higher palmar DA values on the radio-ulnar edges of the Mc1 base $(\mathbf{H} \mathbf{2 C})$. While values average DA values for this species were highest in the radiopalmar region (Fig. 3b) consistent with opposition of the thumb these were only significantly higher than those of bonobos (Fig.5) and DA were also higher at some ulnar landmarks (Fig. 3b). Conversely, humans displayed the highest RBV/TV values at the radial and less extreme palmar region of Mc1 base landmarks, consistent with a habitually and forcefully abducted thumb, flexed in opposition (H2c; Napier, 1956; Halilaj et al., 2014; Feix et al., 2016; D’Agostino et al., 2017, Marchi et al., 2017). These results agree with other studies that have found a palmar concentration of Mc1 base BV/TV in comparison to other apes (Skinner et al., 2015) and other metacarpals (Wong et al., 2018). The radio-palmar concentration found here agrees with that found using the whole-epiphysis method, on a similar sample (Stephens et al., 2018), as well as the fact that osteoarthritis first develops in this region of the Mc1 base (Koff et al., 2003). The present results further refine this radio-palmar RBV/TV distribution to a less markedly palmar subarticular area than that found in other great apes. This pattern may be interpreted as habitual loading of the human TMc joint in a less flexed position than in other extant hominids. This may be a result of the 'screw-home' mechanism of the TMc joint during human opposition where axial rotation of the Mc1 causes the tip of the palmar beak to enter a recess on the trapezium (Edmunds, 2011; D'Agostino et al., 2017). In this position the majority of the Mc1 base is in compression and stabilises the joint without the need of a taut palmar peak ligament but the tip of the beak no longer articulates with the trapezium as it moves ulnarly into this recess (Edmunds, 2011). The more rounded volar beak of $P$. paniscus, for example, would not facilitate this movement as well as the triangular shaped palmar beak of humans (van Leeuwen et al., 2018b). As a result the palmar tip of the human Mc1 base may undergo less loading during habitual forceful opposition of the Mc1 base and so not engender higher RBV/TV values in this region. Conversely non-human great apes that do not habitually perform forceful padto-pad opposition load the most palmar aspect of the Mc1 base during flexion and so have higher RBV/TV values in this region. Proximity patterns of the TMc joint in humans support this interpretation as they demonstrate that the tip of the palmar beak is closer to the trapezium during adduction and during a pad to-side grip than in abduction and during an abducted power grip (Fig.1;D’Agostino et al., 2017b). 


\section{Functional interpretation}

Taken together the current results are consistent with habitual loading of the TMc joint in flexion, and the McP joint in adduction, during frequently used precision and power grips in all non-human great apes studied. The results also suggest that Gorilla uses more varied thumb postures than either Pan or Pongo as they have lower DA at both joints. While locomotion is not the focus of this paper it is of note that the most terrestrial non-human great ape, Gorilla, has a trabecular pattern consistent with loading of the thumb in many positions during manipulation. Not only is this consistent with behavioural observations of mountain gorillas in the wild (Byrne et al., 2001; Neufuss et al., 2018) but also hypotheses that link complex manipulation and tool-use with terrestrial behaviour in general (Meulman, Sanz,Visalberghi \& van Schailk, 2012; Heldstab et al., 2016). Humans, the most terrestrial species studied exhibit a distinct trabecular morphology that suggests a habitually less flexed more abducted TMc joint and a more strongly, or frequently, recruited flexed abducted McP joint than other great apes. This human pattern is consistent with habitual forceful precision grips uniquely practised by humans during manipulation.

While these results appear consistent with what is known of hominid behaviour and anatomy, the function of trabecular bone is not only biomechanical but also physiological, as it is important for mineral homeostasis (Clarke, 2008). Furthermore, trabecular structure may be affected by systemic factors, including the gut biomes of an animal (Tsegai et al., 2018) and is determined genetically to some extent (Lovejoy et al., 2003; Havill et al., 2010; Judex, Zhang, Donahue \& Ozcivici, 2013; Almécija et al., 2015b). Some researchers have also argued that functional adaptation of bone is largely limited to mechanical strains experienced during growth (Bertram and Schwartz, 1991; Lovejoy et al., 2003; Wallace et al., 2017). While developmental signals are certainly present in bone, bone functional adaptation also occurs in the mature skeleton (Ruff et al., 2006) and to some extent during senescence (Homminga et al., 2004). However, it should be noted that the Mc1 head develops from a pseudo-epiphysis whereas the base arises from a true secondary ossification centre (Haines, 1974) reflecting a different evolutionary history from the other metacarpals (Pazzaglia et al., 2018). This developmental difference may also potentially affect trabecular architecture (Lazenby et al., 2011). The functional signal found here, however, appears to be relatively strong, given these potentially confounding variables.

The interpretation of this functional trabecular signal must also consider the shape of the cortical bone which surrounds it as well as soft tissue morphology. It could be argued that the radio-palmar concentration of RBV/TV in humans is due to ulnar deviation of the Mc1 head relative to the base (Drapeau, 2005) in this species rather than an inter-specific difference in manipulation. That is, the trabecular pattern is caused by the same vector of manipulative load in all species but in humans the McP joint load angle differs due to the shape of the Mc1. However, an ulnar trabecular signal is found in the orangutan Mc1 head that is also ulnarly deviated relative to the Mc1 base (Drapeau, 2005) and therefore this signal is likely due to species differences in manipulation. Similarly the size of subarticular Mc1 surfaces (Tocheri et al., 2005) may also affect the current results, since a constant number of landmarks will necessarily sample less subarticular trabeculae of a larger surface or redundantly over-sample a smaller subarticular surface. The use of interpolated trabecular values, however, should ameliorate this sampling effect and it appears to not have affected the relatively large regional patterns found here. Nevertheless, as discussed for the Mc1 head, it would be advantageous to scale DA by trabecular number in future studies to ensure more direct and intuitive 
comparison of species with differently sized bones. Use of a principle trabecular orientation approach (Barak et al., 2017) in future studies may also obviate this complex interpretation.

\section{Conclusion}

In conclusion, while several predictions regarding DA were only partially supported by the data interspecific variation found in the subarticular trabecular architecture of the hominid Mc1 head and base indicates four main findings. Firstly, non-human great apes have an ulnar concentration of RBV/TV values in the subarticular Mc1 head and a palmar concentration in the Mc1 base, consistent with an adducted thumb in pad-to-side grips frequently practiced by these species. Secondly humans have a radial concentration of RBV/TV values in the subarticular Mc1 head and a radio-palmar concentration in the Mc1 base. This statistically distinct pattern in humans is consistent with a habitually abducted thumb used during forceful precision grips, which are employed in many manipulative behaviours, including stone tool production. Thirdly, the low DA found in gorilla Mc1 trabeculae is consistent with more frequent and varied habitual manipulation than has yet been observed in other wild great apes. Finally, this signal is distinct from that of humans, which have significantly higher DA throughout the subarticular Mc1 head, consistent with relatively high loading during flexion and extension of the McP and facilitated by the unique thenar musculature of humans. The use of this comparative sample could aid in the identification of human-like manual behaviours in the hominin fossil record.

\section{Acknowledgements}

We are grateful to Inbal Livne (Powell-Cotton Museum), Anneke van Heteren, Michael Hiermeier (Zoologische Staatssammlung München), Christophe Boesch, Uta Schwarz (MPI-EVA), Ana Ragni (NMNH); Maria Teschler-Nicola and Ronald Muehl (Natural History Museum, Vienna), Jacopo MoggiCecchi and Silvia Bortoluzzi (University of Florence), Frieder Mayer and Christiane Funk (Museum für Naturkunde - Leibniz Institute for Evolution and Biodiversity Science, Berlin) and Birgit Großkopf (Johann-Friedrich-Blumenbach-Institut für Zoologie und Anthropologie der Georg-August-Universität Göttingen) for access to specimens in their care. We also thank David Plotzki (MPI-EVA) and Keturah Smithson (Cambridge Biotomography Centre) for assistance in scanning these specimens, as well as Matthew Tocheri for assistance with landmarking software and Leoni Georgiou for discussions that enhanced this manuscript. We would also like to thank the three anonymous reviewers for their comments that improved this manuscript.This research was supported by European Research Council Starting Grant \#336301, Consolidator Grant \#819960 (CJD, MMS, TLK), the Max Planck Society (MMS, TLK) and the Fyssen Foundation (AB).

\section{Literature cited}

Adams, D. C., L., C. M., Kaliontzopoulou, A., \& Sherratt, E. (2017). Geomorph: Software for geometric morphometric analyses. $\mathrm{R}$ package version 3.0.5. 
Alba, D. M., Moyà-Solà, S., \& Köhler, M. (2003). Morphological affinities of the Australopithecus afarensis hand on the basis of manual proportions and relative thumb length. Journal of Human Evolution, 44(2), 225-254.

Alexander, C. (1994). Utilisation of joint movement range in arboreal primates compared with human subjects: an evolutionary frame for primary osteoarthritis. Annals of the Rheumatic Diseases, 53, 720-725.

Alison, F., \& Badrian, N. (1977). Pygmy chimpanzees. Oryx, 13, 463-468.

Almécija, S., Smaers, J. B., \& Jungers, W. L. (2015). The evolution of human and ape hand proportions. Nature communications, 6.

Almécija, S., Wallace, I. J., Judex, S., Alba, D. M., \& Moyà-Solà, S. (2015b). Comment on "Human-like hand use in Australopithecus africanus". Science, 348(6239), 1101-1101.

Barak, M. M., Lieberman, D. E., \& Hublin, J. J. (2011). A Wolff in sheep's clothing: trabecular bone adaptation in response to changes in joint loading orientation. Bone, 49(6), 1141-1151.

Barak, M. M., Lieberman, D. E., \& Hublin, J. J. (2013b). Of mice, rats and men: Trabecular bone architecture in mammals scales to body mass with negative allometry. Journal of Structural Biology, 183(2), 123-131.

Barak, M. M., Lieberman, D. E., Raichlen, D., Pontzer, H., Warrener, A. G., \& Hublin, J. J. (2013). Trabecular evidence for a human-like gait in Australopithecus africanus. PloS one, 8(11), e77687.

Barak, M. M., Sherratt, E., \& Lieberman, D. E. (2017). Using principal trabecular orientation to differentiate joint loading orientation in the 3rd metacarpal heads of humans and chimpanzees. Journal of Human Evolution, 113, 173-182.

Barakat, M. J., Field, J., \& Taylor, J. (2013). The range of movement of the thumb. Hand, 8(2), 179182.

Bardo, A., Borel, A., Meunier, H., Guéry, J. P., \& Pouydebat, E. (2016). Behavioral and functional strategies during tool use tasks in bonobos. American Journal of Physical Anthropology, 161(1), 125-140.

Bardo, A., Cornette, R., Borel, A., \& Pouydebat, E. (2017). Manual function and performance in humans, gorillas, and orangutans during the same tool use task. American Journal of Physical Anthropology, 164(4), 821-836.

Bardo, A., Vigouroux, L., Kivell, T., \& Pouydebat, E. (2018). The impact of hand proportions on tool grip abilities in humans, great apes and fossil hominins: a biomechanical analysis using musculoskeletal simulation. Journal of Human Evolution, 125, 106-121.

Bertram, J. E., \& Swartz, S. M. (1991). The 'law of bone transformation': a case of crying Wolff? Biological Reviews, 66(3), 245-273. 
Biewener, A. A., Fazzalari, N. L., Konieczynski, D. D., \& Baudinette, R. V. (1996). Adaptive changes in trabecular architecture in relation to functional strain patterns and disuse. Bone, 19(1), 1-8.

Boesch, C., \& Boesch, H. (1993). Different hand postures for pounding nuts with natural hammers by wild chimpanzees. In H. Preuschoft, \& D. Chivers, Eds., Hands of primates (pp. 31-43). Vienna: Springer-Verlag.

Bookstein, F. L. (1991). Morphometric tools for landmark data: geometry and biology. Cambridge, UK: Cambridge University Press.

Breuer, T., Ndoundou-Hockemba, M., \& Fishlock, V. (2005). First observation of tool use in wild gorillas. PLoS Biology, 3(11), e380.

Byrne, R. W., Corp, N., \& Byrne, J. M. (2001). Manual dexterity in the gorilla: bimanual and digit role differentiation in a natural task. Animal Cognition, 4(3-4), 347-361.

Chirchir, H., Zeininger, A., Nakatsukasa, M., Ketcham, R. A., \& Richmond, B. G. (2017). Does trabecular bone structure within the metacarpal heads of primates vary with hand posture? Comptes Rendus Palevol, 16(5-6), 533-544.

Christel, M. (1993). Grasping techniques and hand preferences in Hominoidea. In H. Preuschoft, D. Chivers, Eds., Hands of primates (pp. 91-108). Vienna: Springer.

Christel, M. I., Kitzel, S., \& Niemitz, C. (1998). How precisely do bonobos (Pan paniscus) grasp small objects? International Journal of Primatology, 19(1), 165-194.

Christen, P., \& Müller, R. (2017). In vivo visualisation and quantification of bone resorption and bone formation from time-lapse imaging. Current Osteoporosis Reports, 15(4), 311-317.

Cignoni, P., Callieri, M., Corsini, M., Dellepiane, M., Ganovelli, F., \& Ranzuglia, G. (2008). Meshlab: an open-source mesh processing tool. Eurographics Italian Chapter Conference, 129-136.

Clarke, B. (2008). Normal bone anatomy and physiology. Clinical Journal of the American Society of Nephrology, 3(Supplement 3), S131-S139.

Cooney, W. P., Lucca, M. J., Chao, E. Y., \& Linscheid, R. L. (1981). The kinesiology of the thumb trapeziometacarpal joint. The Journal of Bone and Joint Surgery, 63(9), 1371-1381.

Cooney, W., \& Chao, E. (1977). Biomechanical analysis of static forces in the thumb during hand function. The Journal of Bone and Joint Surgery, 59(1), 27-36.

Cowin, S. C. (1986). Wolff's law of trabecular architecture at remodeling equilibrium. Journal of Biomechanical Engineering, 108(1), 83-88.

Crompton, R. H., Sellers, W. I., \& Thorpe, S. K. (2010). Arboreality, terrestriality and bipedalism. Philosophical Transactions of the Royal Society of London B: Biological Sciences, 365(1556), 3301-331.

Currey, J. D. (2002). Bones: structure and mechanics. Princeton: Princeton University Press. 
D'Agostino, P., Dourthe, B., Kerkhof, F., Stockmans, F., \& Vereecke, E. E. (2017). In vivo kinematics of the thumb during flexion and adduction motion: Evidence for a screw-home mechanism. Journal of Orthopaedic Research, 35(7), 1556-1564.

D'Agostino, P., Dourthe, B., Kerkhof, F., Van Lenthe, G. H., \& Stockmans, F. \&. (2017b). In vivo biomechanical behavior of the trapeziometacarpal joint in healthy and osteoarthritic subjects. Clinical Biomechanics, 49, 119-127

de la Torre, I. (2011). The early Stone age lithic assemblages of Gadeb (Ethiopia) and the Developed Oldowan/early Acheulean in east Africa. Journal of Human Evolution, 60(6), 768-812.

DeSilva, J. M., \& Devlin, M. J. (2012). A comparative study of the trabecular bony architecture of the talus in humans, non-human primates, and Australopithecus. Journal of Human Evolution, 63(3), 536-551.

Diogo, R., Richmond, B. G., \& Wood, B. (2012). Evolution and homologies of primate and modern human hand and forearm muscles, with notes on thumb movements and tool use. Journal of Human Evolution, 63(1), 64-78.

Doran, D. (1996). Comparative positional behavior of the African apes. In W. C. McGrew, L. F. Marchant, and T. Nishida, Eds., Great ape societies (pp. 213-224). Cambridge, UK: Cambridge University Press.

Doran, D. M. (1993). Comparative locomotor behavior of chimpanzees and bonobos: the influence of morphology on locomotion. American Journal of Physical Anthropology, 91(1), 83-98.

Doran, D. M. (1997). Ontogeny of locomotion in mountain gorillas and chimpanzees. Journal of Human Evolution, 32(4), 323-344.

Doube, M., Kłosowski, M. M., Wiktorowicz-Conroy, A. M., Hutchinson, J. R., \& Shefelbine, S. J. (2011). Trabecular bone scales allometrically in mammals and birds. Proceedings of the Royal Society of London B: Biological Sciences, 278(1721), 3067-3073.

Doube, M., Kłosowski, M., Arganda-Carreras, I., Cordelières, F., Dougherty, R., J.S., J., et al. (2010). BoneJ: free and extensible bone image analysis in ImageJ. Bone, 47(6), 1076-1079.

Drapeau, M. S. (2015). Metacarpal torsion in apes, humans, and early Australopithecus: implications for manipulatory abilities. PeerJ, e1311.

Drapeau, M. S., \& Ward, C. V. (2007). Forelimb segment length proportions in extant hominoids and Australopithecus afarensis. American Journal of Physical Anthropology, 132(3), 327-343.

Dunmore, C., Bardo, A., Skinner, M., \& Kivell, T. (2019). Metacarpal trabecular bone varies with distinct hand-positions used in hominid locomotion. Journal of Anatomy, 235(1), 45-66.

Dunn, O. J. (1961). Multiple comparisons among means. Journal of the American Statistical Association, 56, 52-64.

Dunn, O. J. (1964). Multiple comparisons using rank sums. Technometrics, 6, 241-252. 
Edmunds, J. O. (2011). Current concepts of the anatomy of the thumb trapeziometacarpal joint. The Journal of Hand Surgery, 36(1), 170-182.

Faurie, C., Schiefenhvel, W., leBomin, S., Billiard, S., \& Raymond, M. (2005). Variation in the frequency of left-handedness in traditional societies. Current Anthropology, 46(1), 142-147.

Feix, T., Kivell, T. L., Pouydebat, E., \& Dollar, A. M. (2015). Estimating thumb-index finger precision grip and manipulation potential in extant and fossil primates. Journal of the Royal Society Interface, 12(106), e20150176.

Feix, T., Romero, J., Schmiedmayer, H. B., Dollar, A. M., \& Kragic, D. (2016). The grasp taxonomy of human grasp types. IEEE Transactions on Human-Machine Systems, 46(1), 66-77.

Fernández, P. J., Almécija, S., Patel, B. A., Orr, C. M., Tocheri, M. W., \& Jungers, W. L. (2015). Functional aspects of metatarsal head shape in humans, apes, and Old World monkeys. Journal of Human Evolution, 86, 136-146.

Fox, E. A., Sitompul, A. F., \& Van Schaik, C. P. (1999). Intelligent tool use in wild Sumatran orangutans. In S. Parker, R. Mitchel, H. Miles, Eds., The Mentality of gorillas and orangutans (pp. 99-116). Cambridge: Cambridge University Press.

Friston, K. J., Holmes, A. P., Worsley, K. J., Poline, J. P., Frith, C. D., \& Frackowiak, R. S. (1995). Statistical parametric maps in functional imaging: a general linear approach. Human Brain Mapping, 2(4), 189-210.

Frost, H. M. (1987). Bone "mass" and the "mechanostat": a proposal. The Anatomical Record, 219(1), 1-9.

Furuichia, T., Sanzb, C., Koops, K., Sakamakia, T., Ryua, H., Tokuyamaa, N., et al. (2015). Why do wild bonobos not use tools like chimpanzees do? Behaviour, 152, 425-460.

Galletta, L., Stephens, N. B., Bardo, A., Kivell, T. L., \& Marchi, D. (2019). Three-dimensional geometric morphometric analysis of the first metacarpal distal articular surface in humans, great apes and fossil hominins. Journal of Human Evolution, 132, 119-136.

Gross, T., Kivell, T. L., Skinner, M. M., Nguyen, N. H., \& Pahr, D. H. (2014). A CT-image-based framework for the holistic analysis of cortical and trabecular bone morphology. Palaeontologia Electronica, 17(3), 1.

Großkopf, B. (2015). Kulturgeschichtliche und naturwissenschaftliche Ergebnisse zur Ausgrabung des neuzeitlichen katholischen Friedhofes in Götttingen. Göttingen: Göttingen University Press.

Gunz, P., \& Mitteroecker, P. (2013). Semilandmarks: a method for quantifying curves and surfaces. Hystrix, The Italian Journal of Mammalogy, 24(1), 103-109.

Gunz, P., Mitteroecker, P., \& Bookstein, F. L. (2005). Semilandmarks in three dimensions. In D. E. Slice, Ed., Modern Morphometrics in Physical Anthropology (pp. 73-98). Boston, MA: Springer. 
Haines, R. W. (1974). The pseudoepiphysis of the first metacarpal of man. Journal of Anatomy, 117, 145-158.

Halilaj, E., Moore, D. C., Patel, T. K., Laidlaw, D. H., Ladd, A. L., Weiss, A. P., et al. (2014b). Thumb carpometacarpal joint congruence during functional tasks and thumb range-of-motion activities. Engineering in Medicine and Biology Society, 36th Annual International Conference of the IEEE , 4354-4357.

Halilaj, E., Rainbow, M. J., Got, C., Schwartz, J. B., Moore, D. C., Weiss, A. P., et al. (2014). In vivo kinematics of the thumb carpometacarpal joint during three isometric functional tasks. Clinical Orthopaedics and Related Research, 472(4), 1114-1122.

Hamrick, M. W., \& Inouye, S. E. (1995). Thumbs, tools, and early humans. Science, 268(5210), 586587.

Hamrick, M. W., Churchill, S. E., Schmitt, D., \& Hylander, W. L. (1998). EMG of the human flexor pollicis longus muscle: implications for the evolution of hominid tool use. Journal of Human Evolution, 34(2), 123-136.

Harmand, S., Lewis, J., Feibel, C., Lepre, C., Prat, S., Lenoble, A., et al. (2015). 3.3-million-year-old stone tools from Lomekwi 3, West Turkana, Kenya. Nature (521), 310-315.

Haslam, M., Hernandez-Aguilar, A., Ling, V., Carvalho, S., de La Torre, I., DeStefano, A., et al. (2009). Primate archaeology. Nature, 460(7253 ), 339-344.

Havill, L., Allen, M., Bredbenner, T., Burr, D., Nicolella, D., Turner, C., et al. (2010). Heritability of lumbar trabecular bone mechanical properties in baboons. Bone, 46(3), 835-840.

Heldstab, S. A., Kosonen, Z. K., Koski, S. E., Burkart, J. M., Van Schaik, C. P., \& Isler, K. (2016). Manipulation complexity in primates coevolved with brain size and terrestriality. Scientific Reports, e24528.

Hohmann, G., \& Fruth, B. (2003). Culture in bonobos? Between-species and within-species variation in behavior. Current Anthropology, 44(4), 563-571.

Homminga, J., van Rietbergen, B., Lochmüller, E. M., Weinans, H., Eckstein, F., \& Huiskes, R. (2004). The osteoporotic vertebral structure is well adapted to the loads of daily life, but not to infrequent "error" loads. Bone, 34(3), 510-516.

Hunt, K. (1991). Mechanical implications of chimpanzee positional behavior. American Journal of Physical Anthropology, 86(4), 521-536.

Ingmanson, E. J. (1996). Tool-using behavior in wild Pan paniscus: Social and ecological considerations. In A. Russon, K. Bard and S. Parkers, Eds., Reaching into thought: The minds of the great apes (pp. 190-210). Cambridge: Cambridge University Press.

Inouye, S. (1994). Ontogeny of knuckle-walking hand postures in African apes. Journal of Human Evolution, 26(5), 459-485. 
Jacofsky, M. (2009). Comparative muscle moment arms of the primate thumb: Homo, Pan, Pongo, and Papio. (Doctoral Dissertation): Arizona State University.

Johanson, M. E., Valero-Cuevas, F. J., \& Hentz, V. R. (2001). Activation patterns of the thumb muscles during stable and unstable pinch tasks. The Journal of Hand Surgery, 26(4), 698-705.

Jones, E., Oliphant, T., \& Peterson, P. (2001). SciPy: Open Source Scientific Tools for Python. www.scipy.org.

Jones-Engels, L., \& Bard, K. A. (1996). Precision grips in young chimpanzees. American Journal of Primatology, 39(1), 1-15.

Judex, S., Zhang, W., Donahue, L. R., \& Ozcivici, E. (2013). Genetic loci that control the loss and regain of trabecular bone during unloading and reambulation. Journal of Bone and Mineral Research, 28(7), 1537-1549.

Kano, T. (1982). The use of leafy twigs for rain cover by the pygmy chimpanzees of Wamba. Primates, 19, 187-193.

Kazhdan, M., \& Hoppe, H. (2013). Screened poisson surface reconstruction. ACM Transactions on Graphics (ToG), 23(3), 29-42.

Key, A. J., \& Dunmore, C. J. (2015). The evolution of the hominin thumb and the influence exerted by the non-dominant hand during stone tool production. Journal of Human Evolution, 78(1), 6069.

Key, A., Merritt, S., \& Kivell, T. (2018). Hand grip diversity and frequency during the use of Lower Palaeolithic stone cutting-tools. Journal of Human Evolution, 125, 137-158

Kinani, J. F., \& Zimmerman, D. (2015). Tool use for food acquisition in a wild mountain gorilla (Gorilla beringei beringei). American Journal of Primatology, 77(3), 353-357.

Kivell, T. L. (2015). Evidence in hand: recent discoveries and the early evolution of human manual manipulation. Philosophical Transactions of the Royal Society of London B: Biological Sciences, 370(1682), 20150105.

Kivell, T. L., Kibii, J. M., Churchill, S. E., Schmid, P., \& Berger, L. R. (2011). Australopithecus sediba hand demonstrates mosaic evolution of locomotor and manipulative abilities. Science, 333(6048), 1411-1417.

Kivell, T., Deane, A., Tocheri, M., Orr, C., Schmid, P., Hawks, J., et al. (2015). The hand of Homo naledi. Nature Communications, 6.

Koff, M. F., Ugwonali, O. F., Strauch, R. J., Rosenwasser, M. P., Ateshian, G. A., \& Mow, V. C. (2003). Sequential wear patterns of the articular cartilage of the thumb carpometacarpal joint in osteoarthritis. The Journal of Hand Surgery, 28(4), 597-604.

Koops, K. F. (2015). Chimpanzees and bonobos differ in intrinsic motivation for tool use. Scientific Reports, 5, 11356. 
Kraft, T. S., Venkataraman, V. V., \& Dominy, N. J. (2014). A natural history of human tree climbing. Journal of Human Evolution, 71, 105-118.

Lambers, F. M., Koch, K., Kuhn, G., Ruffoni, D., Weigt, C., Schulte, F. A., et al. (2013). Trabecular bone adapts to long-term cyclic loading by increasing stiffness and normalization of dynamic morphometric rates. Bone, 55(2), 325-334.

Landsmeer, J. M. (1962). Power grip and precision handling. Annals of the Rheumatic Diseases, 21(2), 164-170.

Lazenby, R. A., Skinner, M. M., Hublin, J. J., \& Boesch, C. (2011). Metacarpal trabecular architecture variation in the chimpanzee (Pan troglodytes): Evidence for locomotion and tool-use? American Journal of Physical Anthropology, 144(2), 215-225.

Leakey, L. S., Tobias, P. V., \& Napier, J. R. (1964). A new species of the genus Homo from Olduvai Gorge. Nature, 202(4927), 7-9.

Lemelin, P., \& Schmitt, D. (2016). On primitiveness, prehensility, and opposability of the primate hand: the contributions of Frederic Wood Jones and John Russell Napier. In T. Kivell, P. Lemelin, B. Richmond, D. Schmitt, Eds., The Evolution of the Primate Hand (pp. 5-13). New York: Springer.

Lesnik, J. J., Sanz, C. M., \& Morgan, D. B. (2015). The interdigital brace and other grips for termite nest perforation by chimpanzees of the Goualougo Triangle, Republic of Congo. American Journal of Physical Anthropology, 157(2), 252-259.

Lovejoy, C. O., McCollum, M. A., Reno, P. L., \& Rosenman, B. A. (2003). Developmental biology and human evolution. Annual Review of Anthropology, 85-109.

Manduell, K. L., Morrogh-Bernard, H. C., \& Thorpe, S. K. (2011). Locomotor behavior of wild orangutans (Pongo pygmaeus wurmbii) in disturbed peat swamp forest, Sabangau, Central Kalimantan, Indonesia. American Journal of Physical Anthropology, 145(3), 348-359.

Marangoni, A., Belli, M. L., Caramelli, D., Moggi Cecchi, J., Zavattaro, M., \& Manzi, G. (2011). Tierra del Fuego, its ancient inhabitants, and the collections of skeletal remains in the Museum of Anthropology of Florence and Rome. Museologia Scientifica, 5(1-2), 88-96.

Marchi, D., Proctor, D. J., Huston, E., Nicholas, C. L., \& Fischer, F. (2017). Morphological correlates of the first metacarpal proximal articular surface with manipulative capabilities in apes, humans and South African early hominins. Comptes Rendus Palevol, 16(5-6), 645-654.

Marzke, M. W. (1986). Hominid hand use in the Pliocene and Pleistocene: evidence from experimental archaeology and comparative morphology. Journal of Human Evolution, 15(6), 439-460.

Marzke, M. W. (2013). Tool making, hand morphology and fossil hominins. Philosophical Transactions of the Royal Society B: Biological Sciences, 368(1630), 20120414. 
Marzke, M. W., \& Wullstein, K. L. (1996). Chimpanzee and human grips: a new classification with a focus on evolutionary morphology. International Journal of Primatology, 17(1), 117-139.

Marzke, M. W., Marchant, L. F., McGrew, W. C., \& Reece, S. P. (2015). Grips and hand movements of chimpanzees during feeding in Mahale Mountains National P ark, Tanzania. American Journal of Physical Anthropology, 156(3), 317-326.

Marzke, M. W., Marzke, R. F., Linscheid, R. L., Smutz, P., Steinberg, B., Reece, S., et al. (1999). Chimpanzee thumb muscle cross sections, moment arms and potential torques, and comparisons with humans. American Journal of Physical Anthropology, 110(2), 163-178.

Marzke, M. W., Wullstein, K. L., \& Viegas, S. F. (1992). Evolution of the power ("squeeze") grip and its morphological correlates in hominids. American Journal of Physical Anthropology, 89(3), 283-298.

Marzke, M., Tocheri, M., Steinberg, B., Femiani, J., Reece, S., Linscheid, R., et al. (2010). Comparative $3 \mathrm{D}$ quantitative analyses of trapeziometacarpal joint surface curvatures among living catarrhines and fossil hominins. American Journal of Physical Anthropology, 141(1), 38-51.

Marzke, M., Toth, N., Schick, K., Reece, S., Steinberg, B., Hunt, K., et al. (1998). EMG study of hand muscle recruitment during hard hammer percussion manufacture of Oldowan tools. American Journal of Physical Anthropology, 105(3), 315-332.

Matarazzo, S. (2013). Manual pressure distribution patterns of knuckle-walking apes. American Journal of Physical Anthropology, 152(1), 44-50.

Mcclure, N. K., Phillips, A. C., Vogel, E. R., \& Tocheri, M. W. (2012). Unexpected pollex and hallux use in wild Pongo pygmaeus wurmbii. American Journal of Physical Anthropology, 147(Supp.54), 203.

Meulman, E. J., Sanz, C. M., Visalberghi, E., \& van Schaik, C. P. (2012). The role of terrestriality in promoting primate technology. Evolutionary Anthropology: Issues, News, and Reviews, 21(2), 58-68.

Meulman, E. J., \& van Schaik, C. P. (2013). Orangutan tool use and the evolution of technology. In C. Sanz, J. Call, B. C., Eds., Tool use in animals: cognition and ecology (pp. 176-202). Cambridge: Cambridge University Press.

Napier, J. (1961). Prehensility and opposability in the hands of primates. Symposia of the Zoological Society of London, 5, 115-132.

Napier, J. (1962). Fossil hand bones from Olduvai Gorge. Nature, 196, 409-411.

Napier, J. R. (1956). The prehensile movements of the human hand. The Journal of Bone and Joint Surgery. British volume, 38(4), 902-913.

Napier, J. R. (1960). Studies of the hands of living primates. In Proceedings of the Zoological Society of London (pp. 647-657). Oxford: Blackwell Publishing.

Napier, J. R. (1993). Hands. Princeton: Princeton University Press. 
Neufuss, J., Humle, T., Cremaschi, A., \& Kivell, T. L. (2016). Nut-cracking behaviour in wild-born, rehabilitated bonobos (Pan paniscus): a comprehensive study of hand-preference, hand grips and efficiency. American Journal of Primatology, 79(2), 1-16.

Neufuss, J., Robbins, M. M., Baeumer, J., Humle, T., \& Kivell, T. L. (2017). Comparison of hand use and forelimb posture during vertical climbing in mountain gorillas (Gorilla beringei beringei) and chimpanzees (Pan troglodytes). American Journal of Physical Anthropology, 164(4), 651664.

Neufuss, J., Robbins, M., Baeumer, J., Hulme, T., \& Kivell, T. (2018). Manual skills for food processing by mountain gorillas in Bwindi Impenetrable National Park, Uganda. Biological Journal of the Linnean Society, 127(3), 543-562.

Niewoehner, W. A. (2005). A geometric morphometric analysis of Late Pleistocene human metacarpal 1 base shape. In D. E. Slice, Modern Morphometrics in Physical Anthropology (pp. 285-298). Boston, MA: Springer.

Odgaard, A., Kabel, J., van Rietbergen, B., Dalstra, M., \& Huiskes, R. (1997). Fabric and elastic principal directions of cancellous bone are closely related. Journal of Biomechanics, 30(5), 487-495.

Ogihara, N., Kunai, T., \& Nakatsukasa, M. (2005). Muscle dimensions in the chimpanzee hand. Primates, 46(4), 275-280.

Oksanen, J., Blanchet, F., Friendly, M., Kindt, R., Legendre, P., McGlinn, D., et al. (2018). Vegan: Community Ecology Package.

Orr, C. M. (2016). Functional morphology of the primate hand: Recent approaches using biomedical imaging, computer modeling, and engineering methods. In T. Kivell, P. Lemelin, B. Richmond, D. Schmitt, Eds., The Evolution of the Primate Hand. (pp. 227-257). New York: Springer.

Pahr, D. H., \& Zysset, P. K. (2009). From high-resolution CT data to finite element models: development of an integrated modular framework. Computer Methods in Biomechanics and Biomedical Engineering, 12(1), 45-57.

Panger, M. A., Brooks, A. S., Richmond, B. G., \& Wood, B. (2002). Older than the Oldowan? Rethinking the emergence of hominin tool use. Evolutionary Anthropology: Issues, News, and Reviews, 11(6), 235-245.

Paoli, G., Tarli, S. M., Klír, P., Strouhal, E., Tofanelli, S., Del Santo Valli, M. T., et al. (1993). Paleoserology of the Christian population at Sayala (Lower Nubia): an evaluation of the reliability of the results. American Journal of Physical Anthropology, 92(3), 263-272.

Pazzaglia, U. E., Sibilia, V., Casati, L., Salvi, A. G., Minini, A., \& Reguzzoni, M. (2018). The missing segment of the autopod 1st ray: new insights from a morphometric study of the human hand. Journal of Anatomy, 233(6), 828-842. 
Pontzer, H., Lieberman, D. E., Momin, E., Devlin, M. J., Polk, J. D., Hallgrimsson, B., et al. (2006). Trabecular bone in the bird knee responds with high sensitivity to changes in load orientation. Journal of Experimental Biology, 209(1), 57-65.

Pouydebat, E., Gorce, P., Coppens, Y., \& Bels, V. (2009). Biomechanical study of grasping according to the volume of the object: human versus non-human primates. Journal of Biomechanics, 42(3), 266-272.

Pouydebat, E., Laurin, M., Gorce, P., \& Bels, V. (2008). Evolution of grasping among anthropoids. Journal of Evolutionary Biology, 21(6), 1732-1743.

Pouydebat, E., Reghem, E., Borel, A., \& Gorce, P. (2011). Diversity of grip in adults and young humans and chimpanzees (Pan troglodytes). Behavioural Brain Research, 218(1), 21-28.

Preuschoft, H. (1965). Muskeln und gelenk der vorderextremitat des gorillas. Gegenbaurs Morphologisches Jahrbuch, 107, 99-183.

Preutz, J. D., \& Bertolani, P. (2007). Savanna chimpanzees, Pan troglodytes verus, hunt. Current Biology, 17, 412-417.

R Core Team. (2016). R: a language and environment for statistical computing. Vienna.

Rafferrty, K. (1990). The functional and phylogenetir significance of the carpometacarpal joint of the thumb in anthropoid primates. M.A. Dissertation, New York University.

Rein, T. R. (2018). A geometric morphometric examination of hominoid third metacarpal shape and its implications for inferring the precursor to terrestrial bipedalism. The Anatomical Record, 302(6), 983-998.

Reina, N., Cavaignac, E., Trousdale, W. H., Laffosse, J. M., \& Braga, J. (2017). Laterality and grip strength influence hand bone micro-architecture in modern humans, an HR p QCT study. Journal of Anatomy, 230(6), 796-804.

Remis, M. (1995). Effects of body size and social context on the arboreal activities of lowland gorillas in the Central African Republic. American Journal of Physical Anthropology, 97(4), 413-433.

Remis, M. (1998). The gorilla paradox: The effects of body size and hbitat on the positional behavior of lowland and mountain gorillas. In E. Stasser, J. Fleagle, A. Rosenberge, and H. McHenry Eds., Primate Locomotion (pp. 95-106). Boston, MA: Springer.

Richmond, B. G., Roach, N. T., \& Ostrofsky, K. R. (2016). Evolution of the early hominin hand. In T. Kivell, P. Lemelin, B. Richmond, \& D. Schmitt, Eds., The Evolution of the Primate hand (pp. 515-543). New York: Springer.

Rolian, C., Lieberman, D. E., \& Zermeno, J. P. (2011). Hand biomechanics during simulated stone tool use. Journal of Human Evolution, 61(1), 26-41.

Rose, M. (1988). Functional anatomy of the cheiridia. In J. Schwartz, Ed., Orangutan biology. (pp. 299-310). Oxford: Oxfrod University Press. 
Rose, M. D. (1992). Kinematics of the trapezium-1st metacarpal joint in extant anthropoids and Miocene hominoids. Journal of Human Evolution, 22(4-5), 255-266.

Ruff, C. B., \& Runestad, J. A. (1992). Primate limb bone structural adaptations. Annual Review of Anthropology, 407-433.

Ruff, C., Holt, B., \& Trinkaus, E. (2006). Who's afraid of the big bad Wolff?:"Wolff's law" and bone functional adaptation. American Journal of Physical Anthropology, 129(4), 484-498.

Ryan, T. M., \& Shaw, C. N. (2013). Trabecular bone microstructure scales allometrically in the primate humerus and femur. Proceedings of the Royal Society of London B: Biological Sciences, 280(1758), 20130172.

Ryan, T. M., Carlson, K. J., Gordon, A. D., Jablonski, N., Shaw, C. N., \& Stock, J. T. (2018). Human-like hip joint loading in Australopithecus africanus and Paranthropus robustus. Journal of Human Evolution, 121(1), 12-24.

Saers, J. P., Cazorla-Bak, Y., Shaw, C. N., Stock, J. T., \& Ryan, T. M. (2016). Trabecular bone structural variation throughout the human lower limb. Journal of Human Evolution, 97, 97-108.

Samuel, D. S., Nauwelaerts, S., Stevens, J. M., \& Kivell, T. L. (2018). Hand pressures during arboreal locomotion in captive bonobos (Pan paniscus). Journal of Experimental Biology, e170910.

Sarmiento, E. E. (1988). Anatomy of the hominoid wrist joint: its evolutionary and functional implications. International Journal of Primatology, 9(4), 281-345.

Sarringhaus, L. A., Stock, J. T., Marchant, L. F., \& McGrew, W. C. (2005). Bilateral asymmetry in the limb bones of the chimpanzee (Pan troglodytes). American Journal of Physical Anthropology, $128(4), 840-845$.

Scherf, H. T. (2009). A new high-resolution computed tomography (CT) segmentation method for trabecular bone architectural analysis. American Journal of Physical Anthropology, 140(1), 39-51.

Schlager, S. (2017). Morpho and Rvcg-Shape Analysis in R: R-Packages for Geometric Morphometrics, Shape Analysis and Surface Manipulations. In G. Zheng, S. Li and G. Székely, Eds. Statistical shape and deformation analysis: Methods, Implementation and Applications (pp. 217-256). Cambridge, MA: Academic Press.

Skinner, M. M., Stephens, N. B., Tsegai, Z. J., Foote, A. C., Nguyen, N. H., Gross, T., et al. (2015). Human-like hand use in Australopithecus africanus. Science, 347(6220), 395-399.

Skinner, M. M., Stephens, N. B., Tsegai, Z. J., Foote, A. C., Nguyen, N. H., Gross, T., et al. (2015b). Response to comment on "Human-like hand use in Australopithecus africanus". Science, 348(6239), 1101.

Stephens, N. B., Kivell, T. L., Pahr, D. H., Hublin, J. J., \& Skinner, M. M. (2018). Trabecular bone patterning across the human hand. Journal of Human Evolution, 1-23. 
Stephens, N., Kivell, T., Gross, T., Pahr, D., Lazenby, R., Hublin, J., et al. (2016). Trabecular architecture in the thumb of Pan and Homo: implications for investigating hand use, loading, and hand preference in the fossil record. American Journal of Physical Anthropology, 161(4), 603-619.

Strauss, W. (1942). Rudimentary digits in primates. The Quarterly Review of Biology, 17(3), 228-243.

Strouhal, E., \& Jungwirth, J. (1979). Paleogenetics of the late Roman-early Byzantine cemeteries at Sayala, Egyptian Nubia. Journal of Human Evolution, 8(7), 699-703.

Su, A., Wallace, I. J., \& Nakatsukasa, M. (2013). Trabecular bone anisotropy and orientation in an Early Pleistocene hominin talus from East Turkana, Kenya. Journal of Human Evolution, 64(6), 667-677.

Sukhdeo, S., Parsons, J., Niu, X., \& Ryan, T. (2018). Trabecular bone structure in the distal femur of humans, apes, and baboons. The Anatomical Record. (in press) doi.org/10.1002/ar.24050

Susman, R. L. (1979). Comparative and functional morphology of hominoid fingers. American Journal of Physical Anthropology, 50(2), 215-236.

Susman, R. L. (1994). Fossil evidence for early hominid tool use. Science, 265(5178), 1570-1573.

Susman, R. L. (1998). Hand function and tool behavior in early hominids. Journal of Human Evolution, 35(1), 23-46.

Susman, R. L., \& Badrian, A. J. (1980). Locomotor behaviour of Pan paniscus in Zaire. American Journal of Physical Anthropology, 53, 69-80.

Sylvester, A. D., \& Terhune, C. E. (2017). Trabecular mapping: Leveraging geometric morphometrics for analyses of trabecular structure. American Journal of Physical Anthropology, 163(3), 553569.

Thorpe, S. K., \& Crompton, R. H. (2006). Orangutan positional behavior and the nature of arboreal locomotion in Hominoidea. American Journal of Physical Anthropology, 131(3), 384-401.

Tocheri, M. W., Orr, C. M., Jacofsky, M. C., \& Marzke, M. W. (2008). The evolutionary history of the hominin hand since the last common ancestor of Pan and Homo. Journal of Anatomy, 212(4), 544-562.

Tocheri, M. W., Razdan, A., Williams, R. C., \& Marzke, M. W. (2005). A 3D quantitative comparison of trapezium and trapezoid relative articular and nonarticular surface areas in modern humans and great apes. Journal of Human Evolution, 49(5), 570-586.

Toft, R., and Berme, N. (1980). A biomechanical analysis of the joints of the thumb. Journal of Biomechanics, 13(4), 353-360.

Tsegai, Z. J., Kivell, T. L., Gross, T., Nguyen, N. H., Pahr, D. H., Smaers, J. B., et al. (2013). Trabecular bone structure correlates with hand posture and use in hominoids. PLOS ONE, 8(11), e78781. 
Tsegai, Z. J., Skinner, M. M., Pahr, D. H., Hublin, J. J., \& Kivell, T. L. (2018). Systemic patterns of trabecular bone across the human and chimpanzee skeleton. Journal of Anatomy, 232(4), 641-656

Tsegai, Z. J., Stephens, N. B., Treece, G. M., Skinner, M. M., Kivell, T. L., \& Gee, A. H. (2017). Cortical bone mapping: An application to hand and foot bones in hominoids. Comptes Rendus Palevol, 16(5-6), 690-701.

Tuttle, R. H. (1967). Knuckle-walking and the evolution of hominoid hands. American Journal of Physical Anthropology, 26(2), 171-206.

Tuttle, R. H. (1969). Quantitative and functional studies on the hands of the Anthropoidea. I. The Hominoidea. Journal of Morphology, 128(3), 309-363.

Uchiyama, T., Tanizawa, T., Muramatsu, H., Endo, N., Takahashi, H. E., \& Hara, T. (1999). Threedimensional microstructural analysis of human trabecular bone in relation to its mechanical properties. Bone, 25(4), 487-491.

Ulrich, D., Van Rietbergen, B., Laib, A., \& Ruegsegger, P. (1999). The ability of three-dimensional structural indices to reflect mechanical aspects of trabecular bone. Bone, 25(1), 55-60.

van Leeuwen, T., Vanhoof, M. J., Kerkhof, F. D., Stevens, J. M., \& Vereecke, E. E. (2018). Insights into the musculature of the bonobo hand. Journal of Anatomy, 233(3), 328-340.

van Leeuwen, T., Vanneste, M., Kerkof, F., D'Agostino, P., Vanhoof, M., Stevens, J., et al. (2018b). Mobility and structural constraints of the bonobo trapeziometacarpal joint. Biological Journal of the Linnean Society, 127(3), 681-693.

van Schaik, C. P., Fox, E. A., \& Sitompul, A. F. (1996). Manufacture and use of tools in wild Sumatran orangutans. Naturwissenschaften, 83(4), 186-188.

Wallace, I., Demes, B., \& Judex, S. (2017). Ontogenetic and Genetic Influences on Bone's Responsiveness to Mechanical Signals. In C. Percival, J. Richtsmeier, Eds., Building Bones: Bone Formation and Development in Anthropology (pp. 233-253). Cambridge: Cambridge University Press.

Williams-Hatala, E. M., Hatala, K. G., Gordon, M., Key, A., Kasper, M., \& Kivell, T. L. (2018). The manual pressures of stone tool behaviors and their implications for the evolution of the human hand. Journal of Human Evolution, 119(1), 14-26.

Wong, A. L., Meals, C. G., \& Ruff, C. B. (2018). Computed tomographic analysis of the internal structure of the metacarpals and its implications for hand use, pathology, and surgical intervention. Anatomical Science International, 93(2), 231-237.

Wood-Jones, F. (1916). Arboreal Man. London: Edward Arnold.

Wunderlich, R. E., \& Jungers, W. L. (2009). Manual digital pressures during knuckle-walking in chimpanzees (Pan troglodytes). American Journal of Physical Anthropology, 139(3), 394-403. 
Yeh, H. C., \& Wolf, B. S. (1977). Radiographic Anatomical Landmarks of the Metacarpo-Phalangeal Joints. Radiology, 122(2), 353-355.

Zeininger, A., Patel, B. A., Zipfel, B., \& Carlson, K. J. (2016). Trabecular architecture in the StW 352 fossil hominin calcaneus. Journal of Human Evolution, 97(1), 145-158.

Zeininger, A., Richmond, B. G., \& Hartman, G. (2011). Metacarpal head biomechanics: A comparative backscattered electron image analysis of trabecular bone mineral density in Pan troglodytes, Pongo pygmaeus, and Homo sapiens. Journal of Human Evolution, 60(6), 703-710.

Zhou, G.-Q., Pang, Z.-H., Chen, Q.-Q., He, W., Chen, Z.-Q., Chen, L.-L., et al. (2014). Reconstruction of the biomechanical transfer path of femoral head necrosis: A subject-specific finite element investigation. Computers in Biology and Medicine, 52, 96-101.

Zihlman, A. L., Mcfarland, R. K., \& Underwood, C. E. (2011). Functional anatomy and adaptation of male gorillas (Gorilla gorilla gorilla) with comparison to male orangutans (Pongo pygmaeus). The Anatomical Record, 294(11), 1842-1855. 


\section{Figure captions}

Figure 1. Predicted Mc1 positions during a) during full pad-to-pad opposition of the thumb in humans and $\mathbf{b}$ ) in pad-to-side grips in other great apes. In each case a right trapezium, Mc1 and first proximal phalanx (PP1) are depicted in neutral position (white) with the Mc1 (blue) and PP1 (green) superimposed, in the positions thought to be adopted in these grips. Red arrows indicate the movements of the Mc1 and PP1 from neutral to grip position. Inset is a picture of each grip performed in vivo by a human and a gorilla respectively (top; Photograph by: J. Neufuss). The theoretical proximity of the Mc1 to PP1 in these grips is represented by colouring vertices by heat map with red being the closest, and blue being farthest, distance between the articular surfaces (middle). A similar, but measured, pair of proximity maps for the TMc in these grips, is displayed on the Mc1 base in proximal view, palmar aspect up, (bottom; adapted from D'Agostino et al. 2017b).

Figure 2. a) Isosurface model of a Pan troglodytes right first metacarpal, b) Segmented trabecular structure inside cortical shell, c) Diagram of the background grid and one of the spherical VOls at a vertex (purple), d) Volume mesh coloured by BV/TV, shown as a range of $0 \%$ (blue) to $45 \%$ (red), e) Smoothed trabecular surface mesh, f) Surface landmarks on the subarticular head and base (anatomical $=$ red, semi-sliding landmarks on curves=cyan and on surfaces =green), g) RBV/TV interpolated to each surface landmark.

Figure 3. Species Average trabecular values, mapped to average models of a right Mc1 in distal and palmar views of the head as well as a proximal view of the base (left to right) for a) RBV/TV and $\mathbf{b}$ ) DA. (Ggg = Gorilla, $\mathrm{Pt}=$ Pan troglodytes, $\mathrm{Pp}=$ Pan paniscus, $\mathrm{Ppy}=$ Pongo sp., Hs = Homo sapiens).

Figure 4. Significant differences in RBV/TV between species, mapped to average right Mc1 models in palmar (top) and distal (middle) views of the head, as well as a proximal view of the base (bottom). In all views left is ulnar and right is radial. The significantly higher RBV/TV at each landmark is coloured as per species names of that column (Ggg = Gorilla, $\mathrm{Pt}=$ Pan troglodytes, $\mathrm{Pp}=$ Pan paniscus, Ppy = Pongo sp. Hs = Homo sapiens).

Figure 5. Significant differences in DA between species, mapped to average right Mc1 models in palmar (top) and distal (middle) views of the head, as well as a proximal view of the base (bottom). In all views left is ulnar and right is radial. The significantly higher RBV/TV at each landmark is coloured as per species names of that column (Ggg = Gorilla, $\mathrm{Pt}=$ Pan troglodytes, $\mathrm{Pp}=$ Pan paniscus, Ppy $=$ Pongo sp. Hs = Homo sapiens).

Figure 6. PCA plots showing species differences within the first metacarpal head in a) RBV/TV , b) DA values. Each plot shows the first two principle components (PC) in each ray. For RBV/TV PC3 is depicted with PC1, inset, as PC3 explains a non-trivial amount of the variance (14\%) in this case. Landmarks at each extreme of a PC are coloured in grayscale, according to their signed contribution to that PC and plotted on a PC in distal view. White landmarks indicate the highest signed contribution to the PC extreme and black the least.

Figure 7. PCA plots showing species differences within the first metacarpal base in a) RBV/TV, b) DA values. Each plot shows the first two principle components $(P C)$ in each ray and PC3 is depicted with PC1, inset, as PC3 explains a non-trivial amount of the variance ( $14 \%$ and $10 \%$, respectively) in 
each case. Landmarks at each extreme of a PC are coloured in grayscale, according to their signed contribution to that $P C$ and plotted on a PC in proximal view. White landmarks indicate the highest signed contribution to the PC extreme and black the least.

Supporting Information Figure 1. Repeatability tests of landmarks. Each individual first metacarpal head and base was landmarked 5 times on different days. Three individuals of the same species were then subjected to Procrustes transformation in each case. Subsequent permutational omnibus and pairwise MANOVA's were run on the PC1 and PC2 scores, as these cumulatively explained $>85 \%$ of the variation: a) Pan troglodytes heads; b) Pongo heads; c) Pan paniscus heads ; d) Gorilla heads e) Homo sapiens heads; f) Pan troglodytes bases; g) Pongo bases; h) Pan paniscus bases; i) Gorilla bases and j) Homo sapiens bases. All individual specimen repeats were significantly different from each other subsequent to a Bonferroni correction $(p \leq 0.028)$. 